\title{
Enhanced Operations and Maintenance of Pollution Control Equipment
}

\author{
Jearldine I. Northrup \\ Mark D. Ginsberg \\ Joyce C. Baird
}

\begin{abstract}
Laws and regulations mandate that Army installations monitor emissions from industrial processes, and maintain their processes within emissions standards. Army installations commonly use pollution control equipment (PCE) to monitor emissions, and to stay within regulatory and legal limits. Alternatives to use of PCE include using advanced technologies to detect problem conditions, collecting data to predict and determine the cause of failures, using dynamic modeling techniques to model the system or components that have a higher than expected frequency of failure, and verifying the efficacy of the model with data collected from test runs. This study summarized recent attempts to model large systems by using a model based on queuing theory. By studying the methods being used now for reliability centered maintenance (RCM) and the military's predilection to run-to-failure maintenance, this research has produced a linear program from the queuing model that can help reduce equipment downtime. These recent methods may allow U.S. Army installations to optimize maintenance policy for minimal ecological impact.
\end{abstract}

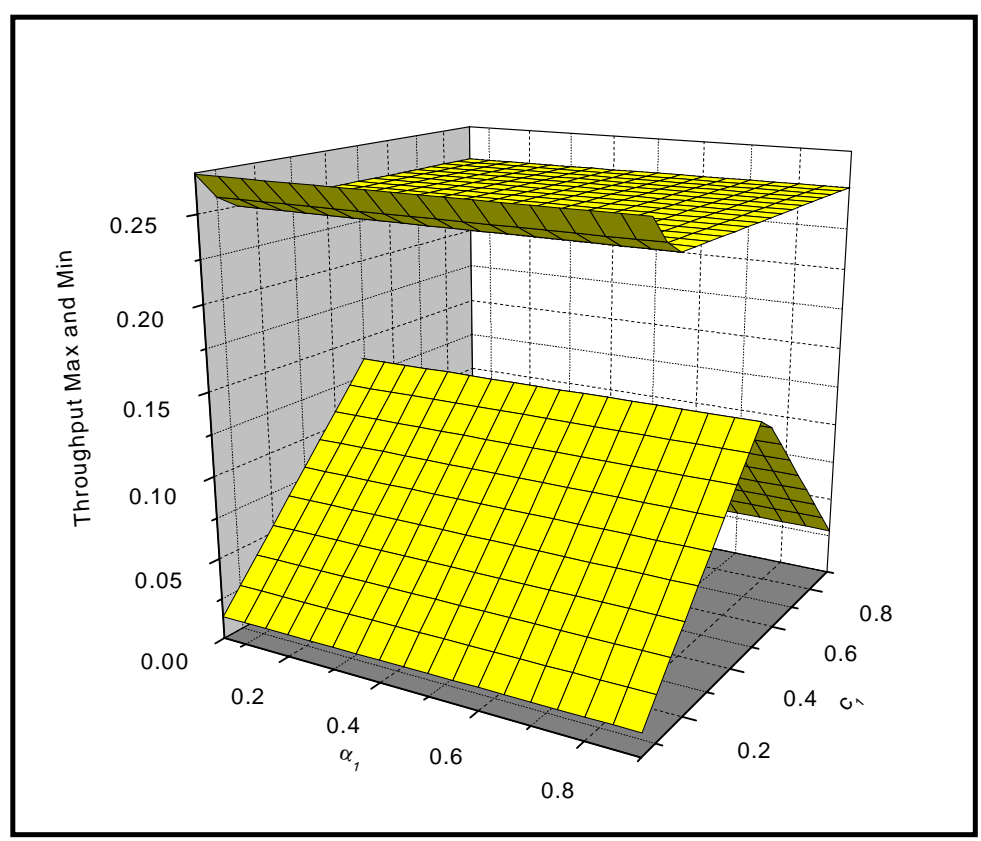




\section{Foreword}

This study was conducted for the Headquarters, Industrial Operations Command (HQIOC), under Project 4A162720D048, "Industrial Operations Pollution Control Technology"; Work Unit U49, "Enhanced O\&M of Pollution Control Equipment." The technical monitor was Chris Vercautren, HQIOC-EQC.

This work was performed by the Environmental Processes (CN-E) Branch of the Installations (CN) Division, U.S. Army Construction Engineering Research Laboratory (CERL). The CERL principal investigator was J earldine I. N orthrup. J erry Benson is Branch Chief, CECER-CN-E, and Dr. J ohn Bandy is Division Chief, CECER-CN. The technical editor was William J. Wolfe, Information Technology Laboratory.

The Director of CERL is Dr. Michael J . O'Connor.

\section{DISCLAIMER}

The contents of this report are not to be used for advertising, publication, or promotional purposes. Citation of trade names does not constitute an official endorsement or approval of the use of such commercial products. All product names and trademarks cited are the property of their respective owners.

The findings of this report are not to be construed as an official Department of the Army position unless so designated by other authorized documents.

DESTROY THIS REPORT WHEN IT IS NO LONGER NEEDED. DO NOT RETURN IT TO THE ORIGINATOR. 


\section{Contents}

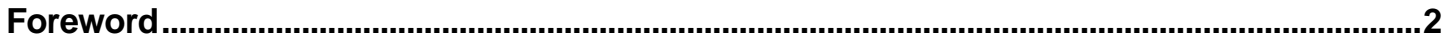

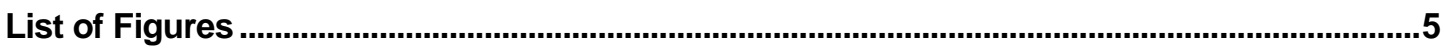

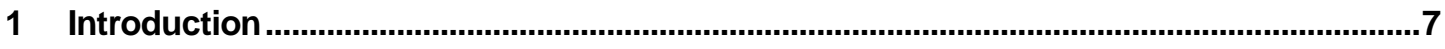

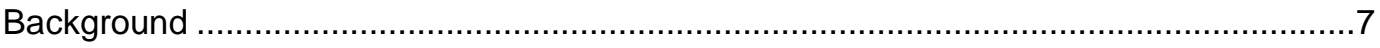

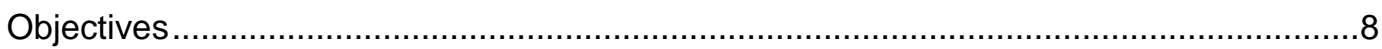

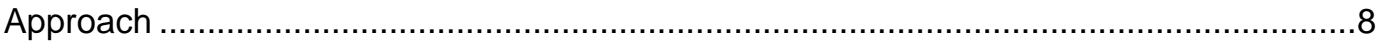

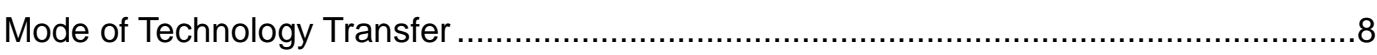

2 Dynamic Modeling - A Technique for Operation and Maintenance of Pollution

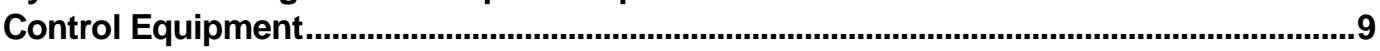

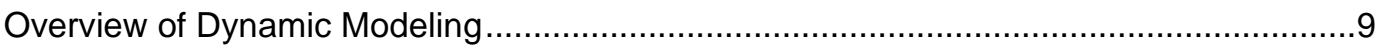

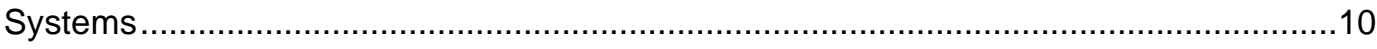

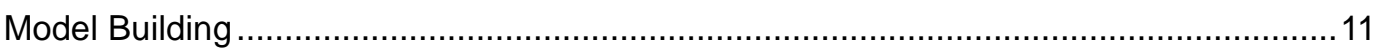

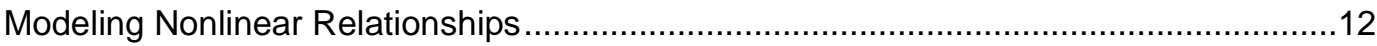

3 Advanced Technologies for Operation and Maintenance of Pollution Control

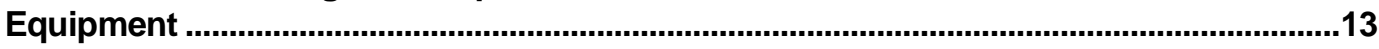

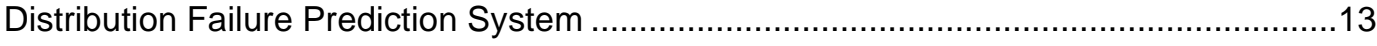

Object-Oriented Cognitive Decision Support Engine ................................................14

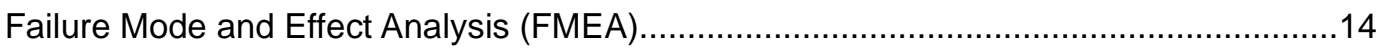

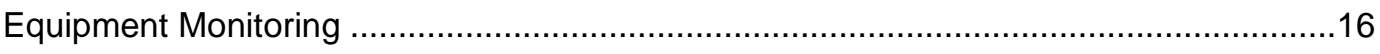

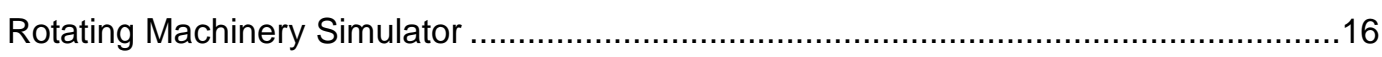

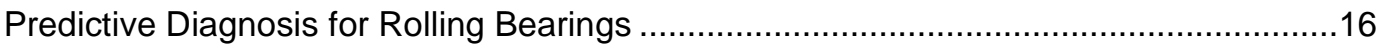

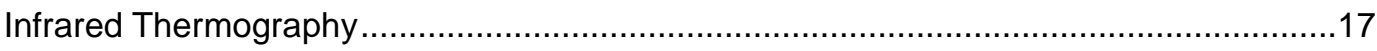

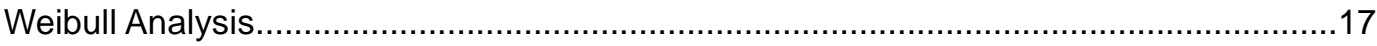

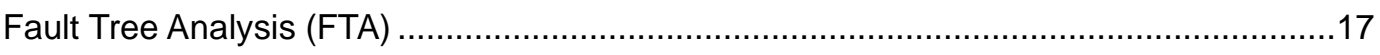

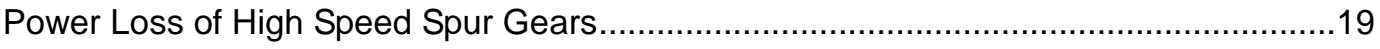

Artificial Neural Network (ANN) and Condition Monitoring...........................................19

4 Case Studies Using Advanced Technologies for Operation and Maintenance of

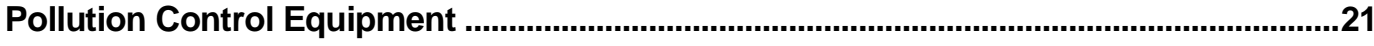

Vibration Interpretation Using Simulation and the Intelligence of Networks

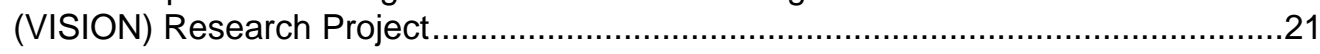

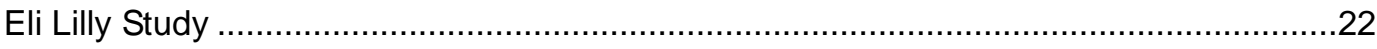

General Electric (GE) Study of Primary Coiling Bearing Run-In Equipment....................23 


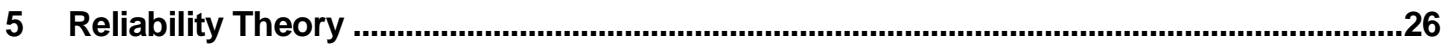

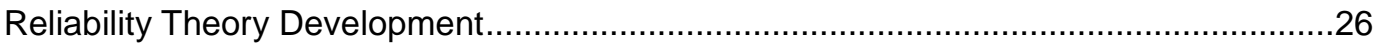

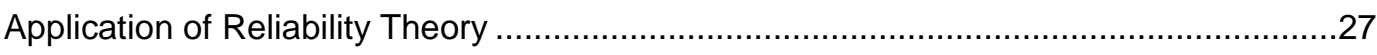

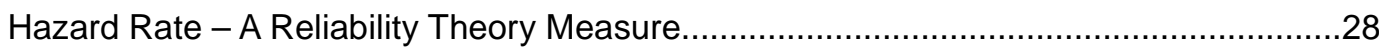

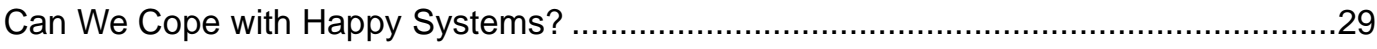

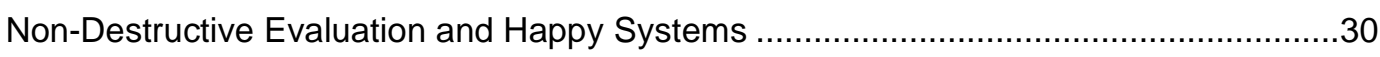

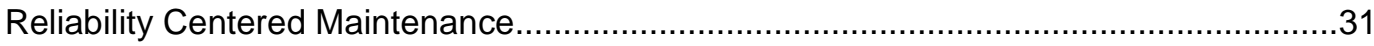

Harmonizing Reliability Centered Maintenance with Army Policy..................................31

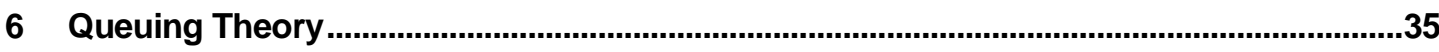

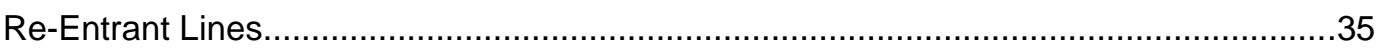

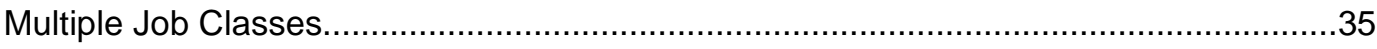

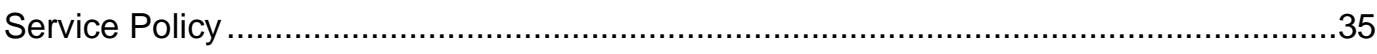

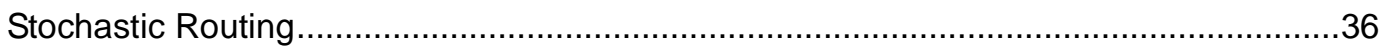

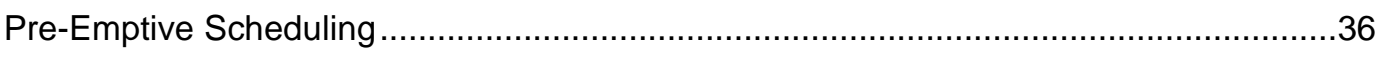

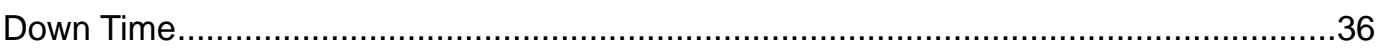

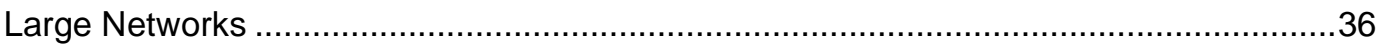

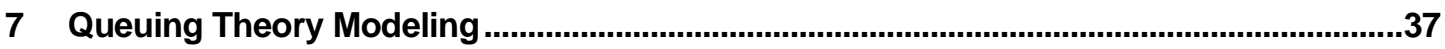

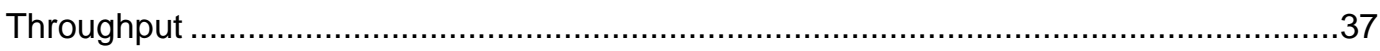

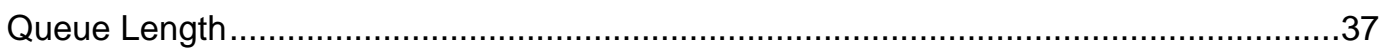

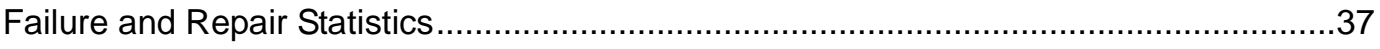

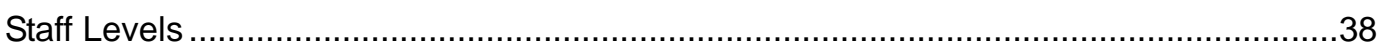

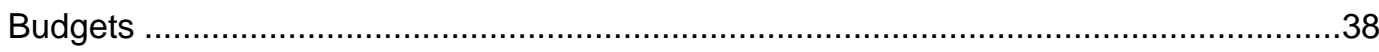

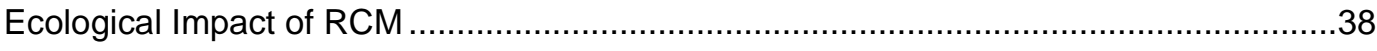

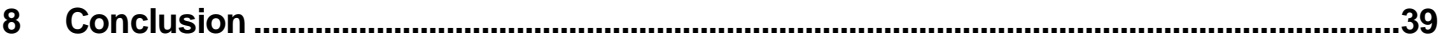

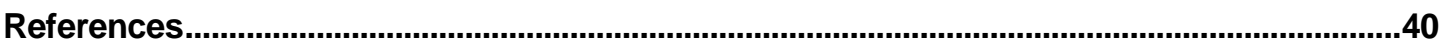

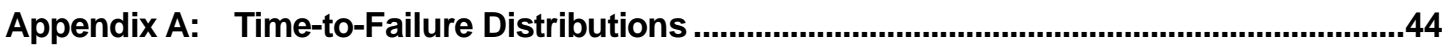

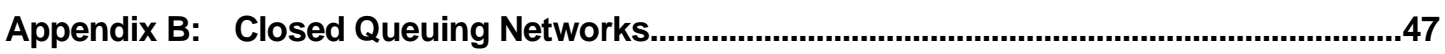

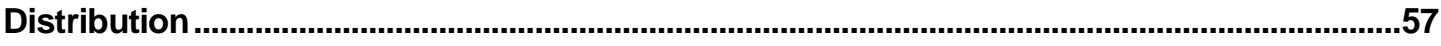

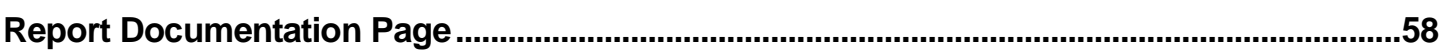




\section{List of Figures}

\section{Figures}

1 The process of model construction (Hannon and Matthias 1994, p 4) ..................10

2 Block diagram of distribution failure prediction system (adapted from System Block Diagram. http://www.keyin.co.kr/eng/Product/1/d 7.html) .........................13

3 The structure of a neural network. (http://www.zsolutions.com/light.htm) .............19

4 Inside a hidden node. (http://www.zsolutions.com/light.htm) .............................20

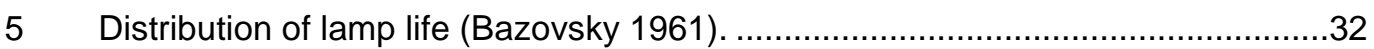

$6 \quad$ Wearout curves of three lamp generations (Bazovsky 1961)..............................33

$7 \quad$ Stabilization of failure frequency (Bazovsky 1961) ..............................................33

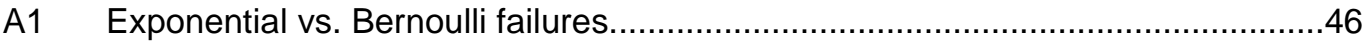

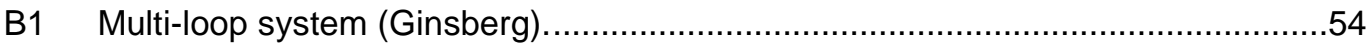

B2 Throughput as a function of loop fraction population and weighting vector (Ginsberg)

B3 Lower bound asymptotic loss as a function of loop fraction population and weighting vector (Ginsberg).

B4 Upper bound asymptotic loss as a function of loop fraction population and weighting vector (Ginsberg).

B5 Theoretical bounds and simulation of throughput as a function of total population (Ginsberg).

B6 Summary of throughputs (Ginsberg). 



\section{Introduction}

\section{Background}

Laws and regulations mandate that Army installations monitor emissions from industrial processes, and maintain their processes within emissions standards. Failure to follow regulations may result in health and safety hazards to installation employees and persons living in the surrounding areas, and may incur heavy fines for the installations. Army installations that have industrial operations commonly use pollution control equipment (PCE) to monitor emissions, and to stay within regulatory and legal limits. PCE has become an integral part of manufacturing systems.

Nevertheless, PCE is no "easy cure" for all problems of hazardous emissions. PCE must be carefully selected and used only in those applications for which it was designed. Users must take care that PCE is well matched when it is simply "added-on" to an established piece of machinery. Also, PCE, like any other complex machinery, requires maintenance for optimal performance. If PCE is not carefully operated and maintained, emissions from manufacturing processes may exceed limits and cause environmental hazards. Moreover, PCE maintenance is expensive and labor intensive, and often is not a high priority item in a manufacturing facility.

Before investing dollars in expensive sensors and maintenance programs, manufacturing installations should look at other available options. Options include using advanced technologies to detect problem conditions, collecting data to predict and determine the cause of failures, using dynamic modeling techniques to model the system or components that have a higher than expected frequency of failure, and verifying the efficacy of the model with data collected from test runs.

An earlier CERL publication (Northrup et al., September 1998) discussed problems of design and their flaws and the problems associated with manufacturers' statistical analysis for failure mode of manufacturing. Other previous CERL work (Chalifoux, Northrup, and Baird 1999; Chalifoux, Northrup, and Chan 1999) have shown that Reliability Centered Maintenance (RCM) usually depends upon test regimens rather than approaching the subject from a statistical viewpoint even though statistics have been used in manufacturing quite successfully. 
Statistical maintenance modeling, even when approached from different initial viewpoints, reveals the impact of different maintenance policies. Until recently, large scale systems have escaped effective analysis. This study attempted to model large systems by using a model based on queuing theory, to produce a model that can reduce equipment downtime and help optimize maintenance policy for minimal ecological impact.

\section{Objectives}

The objectives of the project were to investigate dynamic modeling and advanced maintenance technologies and the use of these technologies in detecting systemic problem areas. Another objective was to develop a dynamic computer model based on queuing theory and using off-the-shelf software to predict and analyze failure distribution in systems.

\section{Approach}

Advanced maintenance technologies and techniques for use in detecting problem conditions in industrial systems were studied. These technologies and techniques include dynamic modeling, acoustic emission methods, Failure Modes and Effects Analysis (FMEA), spectrum and waveform analysis, ultrasound, infrared thermography, and vibration monitoring. Reliability Theorem (RT) and its application to RCM, including a newer approach to RCM from queuing analysis, provided the basis for developing a computer model based on queuing theory to apply to complex systems.

\section{Mode of Technology Transfer}

It is anticipated that the queuing theory computer model and documentation will be available on the CERL web page, available at URL: 


\section{Dynamic Modeling - A Technique for Operation and Maintenance of Pollution Control Equipment}

\section{Overview of Dynamic Modeling}

Researchers use models to explain real-world situations. Models begin as abstract ideas about reality. To implement a model, one must examine the assumptions underlying the abstract ideas used to create the model. Models allow us to explain and sometimes predict the outcomes of the structural and dynamic assumptions that one makes in abstraction. Developing a model can be a complicated procedure, but the process can be simplified by identifying a set of general procedures. Figure 1 shows a simplified form of these general procedures. Sometimes real events cause us to look at particulars of these events, and in turn, these particular interests may be restated as a set of questions regarding the events and what brought them about. By identifying key elements of processes and observations, we can form an abstraction of the real events. These key elements include both the variables that describe the events and the relationships among the variables. Ultimately, both the variables and their relationships establish the model's structure. We can then use the model to formulate conclusions and predict the outcome of future events. Comparing conclusions and predications to real events may reveal that a model is inaccurate, acceptable, or needs revisions. Model building is a continuum of revisions, comparisons, and changes that all lead to a better understanding of the reality in question.

Models may represent a specific phenomenon at a single point in time, such as the location and size of a city, or they may represent rates of change over time, such as the rate of migration to or from a city. The latter type of model is a "dynamic model." The present study used the principles of dynamic modeling to construct computer models to predict PCE maintenance. Computer models help to clarify real-world processes because computer simulation can be applied to imitate the actual forces presumed to cause a system's behavior. 


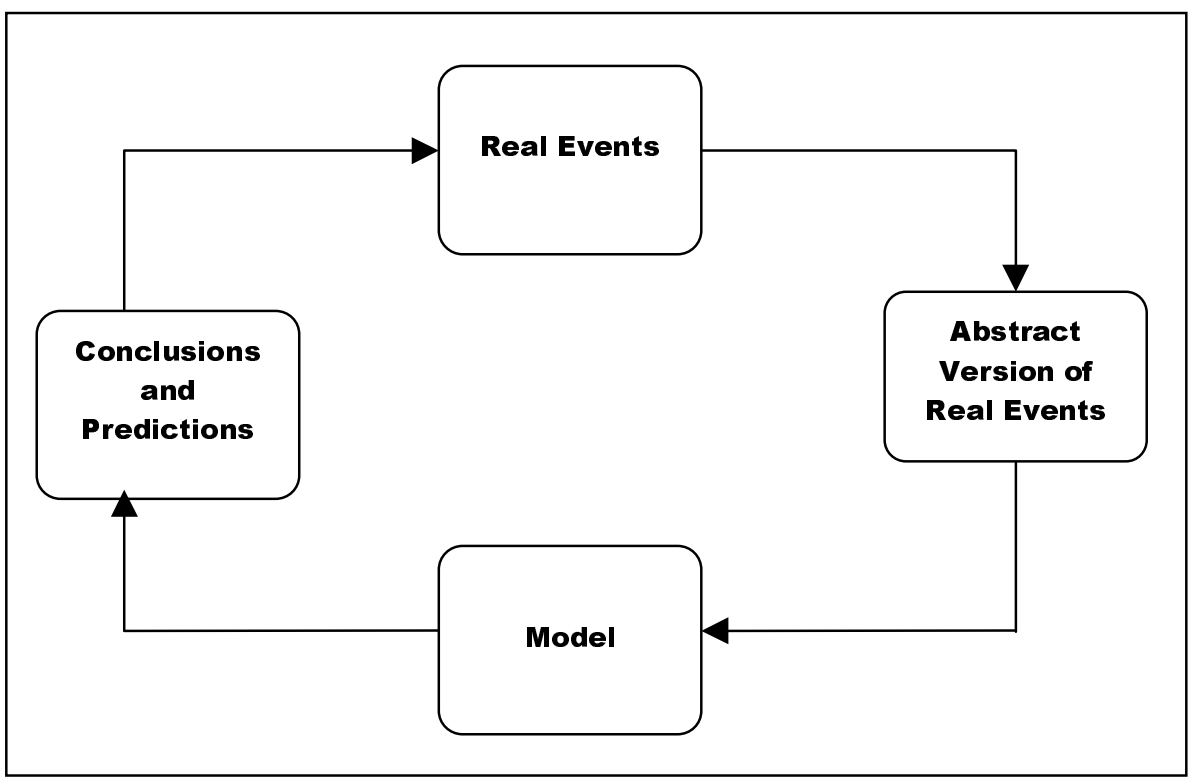

Figure 1. The process of model construction (Hannon and Matthias 1994, p 4).

Initially, models should be kept simple. They may otherwise exceed the complexity of the real-world system that they were meant to explain. Complexity may be added later if the initial model does not produce the real effects. Models are causal because they are built from general rules that demonstrate how each element in a system responds to changes of other elements. A model is a device that keeps us organized during data gathering and evaluating knowledge about the mechanisms that lead to changes in a system.

\section{Systems}

Before developing a model of a system, it is important to understand some aspects of systems. Systems include el ements called variables. These are further described as state and control variables. State variables may be conserved or nonconserved. Conserved state variables denote an accumulation of materials or information, such as population. Nonconserved state variables are indicators of some part of a system's condition. Examples of nonconserved state variables are price and temperature.

The elements in a system that represent changes in state variables are control variables or flows, and they are responsible for updating state variables. The "number of barrels of waste extracted per period" is a control variable as it changes the state variable "reserves of waste." 
Another aspect of a system is the interaction of components within the system, in the form of feedback, both positive and negative. Feedback results from changes in a system component that cause changes in other components. Ultimately, these latter changes affect the component that originally initiated the change. If the series of changes strengthen the original process, then the feedback is positive. On the other hand, if the original change is counteracted by the series of changes, then the feedback is negative. Positive feedback processes magnify disturbance and move the system away from equilibrium. Negative feedback processes by counteracting disturbances lead a system toward steady state.

\section{Model Building}

According to Hannon and Matthias (1994, p 7), the model building process includes a series of steps:

1. Define problem and goals. Carefully structure the questions regarding the problem you require the model to answer. Decide whether the goals of the model are to be descriptive or predictive.

2. Designatethestatevariables. Keep this step simple and denote units for the variables.

3. Select control variables. Choose control variables and corresponding flow controls into and out of the state variables. Record which state variables are donors and which are reci pients in relation to the control variables. Also, note the control variable units. At this step, use one type of control to represent a dass of similar controls.

4. Sel ect parameters for thecontrol variables. When selecting parameters for control variables, ensure that you know to which function the controls and their parameters relate. Note the units for parameters.

5. Check theresulting mode. Check the resulting model for violations of laws, continuity requirements, and consistency of units.

6. Sehow themodel will work. Choose the following: a time horizon, which you will use to look at the dynamic behavior of the model, the duration of each time interval for the updating of state variables, and the procedure for calculating flows. Using a graph, estimate the variation of the state variable curves.

7. Run themode. Choose different lengths for each time interval and alternate the integration procedures to see if the results are the same.

8. Vary the parameters. Vary the parameters to make sure the graph still makes sense. Revise the model to incorporate revisions to errors and irregularities. 
9. Comparetheresults to experimental data. To do this, you may have to close off sections of the model so that you can simulate a laboratory experiment.

10. Revise the parameters. Revise parameters to include exceptions to the experimental results and to increase the complexity of the model, if necessary.

\section{Modeling Nonlinear Relationships}

Linearity refers to lines, planes, and (flat) three-dimensional space, and these objects always appear the same from any aspect. A nonlinear object such as a sphere appears different on different scales. When it is viewed up close, it appears as a plane, whereas from a distance it looks like a point. Nonlinear relationships occur when a control variable does not depend linearly on other variables, but for example, varies with the square root of another variable.

Nonlinearities are especially important in developing models, as many real systems are ruled by nonlinearities. Usually, nonlinear systems do not have specific mathematical solutions and often include characteristics that were not expected or that were incorrectly identified. These unexpected characteristics include chaos. In mathematical terms, chaos is unpredictable long time behavior that arises in a deterministic dynamical system, due to sensitivity to initial conditions. A dynamical system is one that has a state space, whose coordinates explain its dynamical state at any instant of time. A dynamical system possesses also a dynamical rule that specifies the imminent future trend of all state variables from the present values of the same state variables. Dynamical systems may be deterministic or stochastic. Most nonlinear science deals with deterministic systems. A dynamical system is deterministic if a unique resultant to each state exists, and a dynamical system is stochastic if more than one resultant selected from a probability distribution exists. Dynamical systems can also have discrete or continuous time.

In discrete event models, there are events and specific time intervals between the events. The occurrence of events drives the model in discrete event models. Computer models of physical systems essentially are discrete approximations where a series of discrete events represents changes in system state. On the other hand, time moves forward at regular intervals; there is a direct relationship between processes and time, in continuous models. Deterministic differential equations and algebraic equations are required to describe continuous simulation models. 


\section{Advanced Technologies for Operation and Maintenance of Pollution Control Equipment}

The researchers for this project studied advanced technologies available on the commercial market. Although many of these individual technologies are applicable to solve operations and maintenance problems at industrial installations, due to constraints of both budgets and personnel in the military we did not propose their purchase and use. Instead we used the knowledge of the technologies only as a basis to advance our research of cyclic problems in undefined failure modes. The following examples, therefore, are included as reference material for those who may want to follow up on our investigations.

\section{Distribution Failure Prediction System}

This state-of-the-art system is designed to detect failure symptoms and incipient failures in distribution feeders. It allows the user to perform diagnosis and condition monitoring in distribution lines. By preventing distribution failures, power quality improves and optimal equipment operation is achieved. Figure 2 shows a block diagram of the distribution failure prediction system.

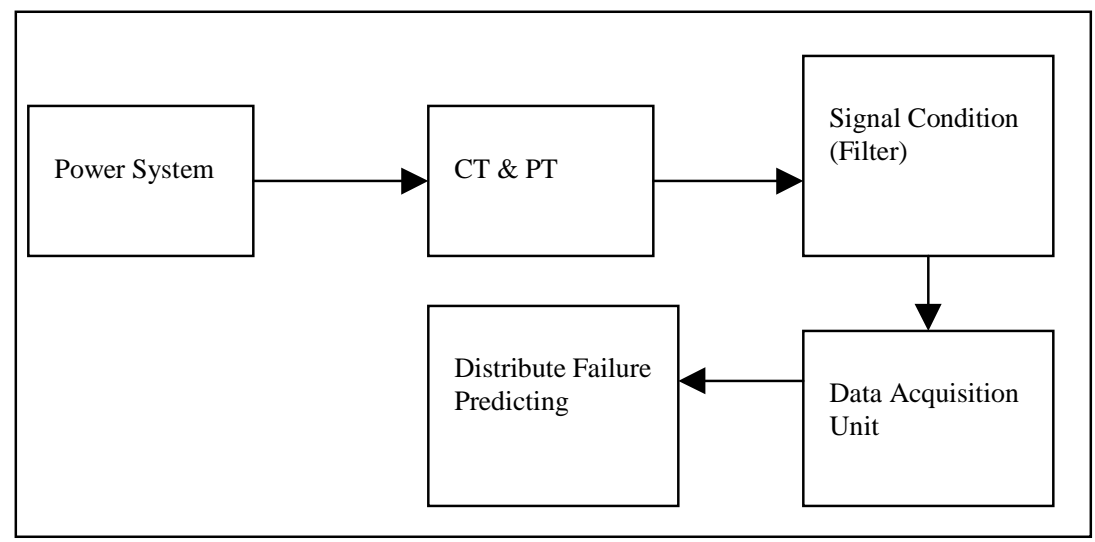

Figure 2. Block diagram of distribution failure prediction system (adapted from System Block Diagram. http://www.keyin.co.kr/eng/Product/1/d 7.html) 
Features of the system include:

- an expert system that used artificial intelligent language

- an ability to predict the insulator failure

- intelligent decisionmaking using frequency parameters.

\section{Object-Oriented Cognitive Decision Support Engine}

This system searches for data and returns the sources that are of particular interest to the decisionmakers' problem. The system uses a "smart scout" to track and identify data from static and dynamic databases, real-time instruments, images (visual, radar, satellite), and direct input. It then notifies the decisionmaker of changes in the data that affect the decision.

This engine includes the following capabilities:

- It manages the cognitive process and data mining operations through intelligent agents.

- It devel ops data "scouts" that look at data sources and report changes.

- It improves decisions based on past performance.

- It allows changes in information that are reflected immediately in decisions.

- It consolidates information from multiple distributed sources for human action.

Source: http://www.nasec.ctc.com/manuknow/technica.htm

\section{Failure Mode and Effect Analysis (FMEA)}

This is a technique used to identify and eliminate known or potential problems from a system. FMEA should be integrated into the initial design review, and it should be an ongoing process throughout the life of the product.

Failure mode is a function of a part number. In a given system, each component part number is analyzed to ascertain possible failure modes, for example, open, short, mechanical failure, etc. Theoretically, each part has limitless potential failure modes, but in reality there is a point of diminishing returns where the cost added exceeds the derived benefits. Failure modes that have the same effect may be combined and separated later if necessary. Initially, FMEA should include all system components that would be repaired or replaced during a maintenance activity, and other failure modes may be added as failures occur. 
The effect of a part failure relies on how the part functions in the system. Even though two valves have the same part number, the effect of a failure rests on what each valve is governing. It is therefore imperative that each component in a system have a unique symbol independent of the part number.

The relative importance of a failure mode is denoted by its RPN number, which is cal culated from the formula:

$$
R P N=S * O * D
$$

Eq. 1

where:

$\mathrm{S}=$ the severity of a potential failure, which is assigned a value from 1 to 10 , where 10 is the most severe failure.

$\mathrm{O}=$ the occurrence of the failure (Relative F ailure Rate) which is assigned a value from 1 to 10 , where 10 is the highest failure rate.

$D=$ the ability to detect a failure, which is assigned a value from 1 to 10 , where 10 is the most difficult to detect.

The fact that FMEA has not been widely used is due, according to discussions between engineers and researchers J eong and I izuka, to the following:

1. FMEA is a time-consuming technique that gives unsatisfactory results.

2. The prediction of failure mode depends too much on a predictor's experience and organizational information. Hence, failure mode omission will result.

3. Evaluation of the seriousness of the failure mode is difficult.

4. The experience gained from an FMEA is difficult to reuse.

In response to the above criticisms of FMEA, J eong and lizuka proposed a technique to prevent the above difficulties with FMEA. First, they investigated problems associated with failure mode prediction and suggested a method to predict failure modes effectively. J eong and lizuka proposed three approaches to effectively predicting failure modes with less omission: (1) failure mode prediction based on "association" (Yeong and lizuka 1996), (2) preparation of Failure Mode Mechanism (FMM) diagram (Yeong and Iizuka 1997), and (3) failure mode prediction based on "hierarchy" (Yeong and lizuka 1996). Second, they presented a method to analyze the cause and effect of failure modes effectively. Third, they applied these methods to refine the above proposals, to ensure the effective application of FMEA. 


\section{Equipment Monitoring}

Tracor, whose customers include the U.S. Navy, the National Aeronautics and Space Administration (NASA), ARPA, and several oil companies, defines six different monitoring functions as follows: (1) continuous protection against catastrophic failure, (2) early detection of machine abnormalities, (3) accurate diagnosis of problem, (4) assessment of level severity, (5) accurate prediction of future machine condition versus time (including time to failure), and (6) generation of feedback information for control of machine operational characteristics. Each of these functions, although requiring different monitoring system design, begins with fault mode selection and data collection.

\section{Rotating Machinery Simulator}

This technology is designed as an educational tool for the study of vibration due to rotating machinery. It allows an operator to learn the principles of rotating machinery in a controlled environment. The main features of a commercially available rotating machinery vibration training tool are:

- rotating machinery vibration simulation

- multi-planebalancing

- dynamically induced structural vibration

- sixteen weight positions per plane

- two $100 \mathrm{mV} / \mathrm{g}$ accelerometers

- Magnetic tachometer for $1 /$ rev signal

- 50 piece balance weight kit with hex keys

- Optional configuration settings for popular balancing equipment

Source: http://altasol.com/rms01.htm

\section{Predictive Diagnosis for Rolling Bearings}

Although many rolling bearings are used in a mechanical plant, failure of just one bearing can result in a total shutdown. Acoustic emission (AE) methods have shown great promise in the successful prediction of fatigue in rolling bearings. Analysis of the AE signal also yields information regarding fatigue crack propagation.

Source: http://www.mel.go.jp/mainlab/kiso/kis0le.html 


\section{Infrared Thermography}

Infrared thermography is a technology that is used to survey machines and structures to detect problems. Operators employ portable infrared cameras to convert thermal energy into high-resolution images for quantitative temperature analysis. The images are collected in minutes and problems are immediately identified. The images can be stored on a computer and used for trending in ensuing surveys. Applications of infrared thermography include the following:

- Electrical and mechanical maintenance.

- Easy detection of overheating of bearings, switchgear, transformers, busbars, overhead power lines and substations.

- Quick recognition of faulty components in el ectrical equipment.

- In metal refining, smelting or sintering processes, refractory wear can be identified in pots, kilns, furnaces, ladles, and torpedo cars.

- In buildings and cold storage units, insulation of boilers, pipework and steamtraps, the integrity of cladding can be easily monitored.

Source: http://www.ozemail.com.au/-its3d/thermog.html

\section{Weibull Analysis}

The Weibull distribution analysis can be used to predict failure rates as well as to describe the failure of parts and equipment. The Weibull analysis provides information on:

- characteristic life

- standard deviation of life

- mean life

- reliability functions

- reliablelife

- median life initial failure rate per unit time.

Source: http://www.bassengineering.com/weibull.htm

\section{Fault Tree Analysis (FTA)}

FTA is an analytical technique that tries to combine all of the factors that affect the success or failure of a product, process, or mission into a single FTA Logic Diagram. A single FTA Logic Diagram uses symbols called "Logic Gates," which are similar to the symbols used by electronic circuit designers. The FTA Logic 
Diagram proves to be a sound method to define the relationships between the hardware, software, and human components of a system.

The inputs to a Logic Gate (symbol) depict the status of a part and/or other factor that is being included in the analysis. The output from a Logic Gate (symbol) is a logic state that represents a condition existing in a system. When the output from a Logic Gate changes, an event occurs.

The state is TRUE if a part or other factor is functioning correctly. If the logic statement is TRUE, we assign to it a Boolean logic value of one (1). On the other hand, the state is FALSE if the part or other factor is malfunctioning. In this case, we assign to it a Boolean logic value of zero (0).

A F ault Tree Analysis is actually performed by determining what occurs in a system when the status of a part or other factor changes. There is a minimum criterion for success, which is that one single failure cannot cause injury or an undetected loss of control over the process. In the case where extreme hazards exist or during the processing of a highly valued product, the criterion may be augmented to require toleration of multiple failures.

An FTA considers both positive and negative events. Logic tree segments that lead to a negative event, an accident, for example, define all of the elements that could go wrong to cause the negative event. The logic tree segments for negative events are apt to use more OR gates than AND gates, with the exception of redundant safeguards. Logic tree segments that lead to a positive event define everything that works together for the machine to operate. Logic trees for positive events, for example maintenance troubleshooting trees, in general use more AND gates than OR gates, with the exception of redundancy.

NAND and NOR gates primarily define countermeasures that, if true, allow the system to tolerate conditions that ordinarily result in safety hazards or machine failure. For more information on Boolean or logic functions and logic gates, access the following web site:

Sources:

http://www.bassengineering.com/FTA.htm http://gatsby.lit.tas.edu.au/tibs/mprinc/logicb.html 


\section{Power Loss of High Speed Spur Gears}

Power loss of high speed gears in industrial machinery leads to elevation of the temperature of the gears and their lubricant. Research showed the relationship between power loss and the mechanism of heat occurrence in an effort to create a gear transmission system of higher efficiency. Researchers measured the rising temperatures of the lubricant and gears that is converted into power losses of the gears. They analyzed the sources and characteristics of power loss by changing gear speed, tooth load, oil flow rate, and other running conditions of gears. The researchers correlated characteristics of power loss sources to the gear tooth form and other gear design parameters to reduce power loss.

Source: http://www.mel.go.jp/mainlab/kiso/kis02e.html

\section{Artificial Neural Network (ANN) and Condition Monitoring}

In an ideal world, condition monitoring of a complex el ectromechanical plant requires the skilled personnel with knowledge of these systems. However, with the increasing depletion of such resources, ANN may offer a suitable alternative.

Neural networks use a set of processing elements or nodes that are analogous to neurons in the brain. The elements are interconnected in a network that has the ability to identify patterns in data as the network is exposed to the data. Figure 3 shows a schematic of a neural network.

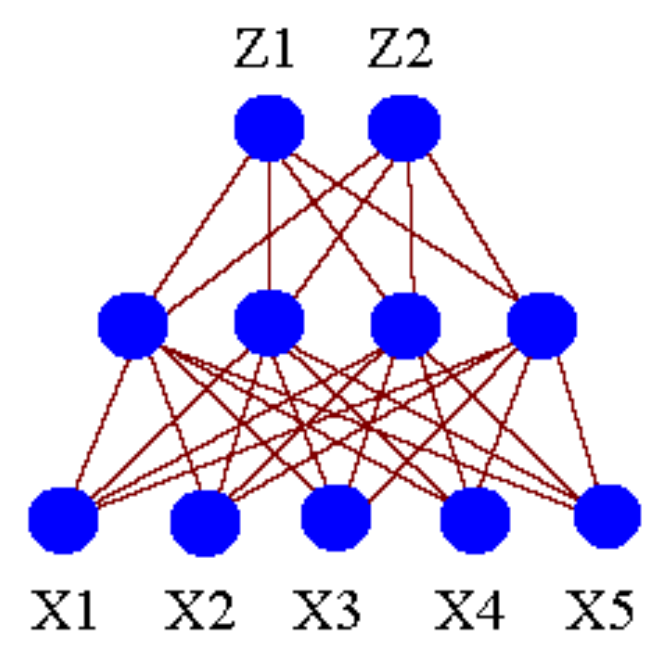

Figure 3. The structure of a neural network. (http://www.zsolutions.com/light.htm) 
In Figure 3, the bottom layer corresponds to the input layer, with 5 inputs $X 1$ through X5. The middle layer, which performs most of the work, is known as the "hidden layer" and contains a variable number of nodes. In this figure, the output layer has two nodes, Z1 and Z2, that represent the output values to be determined from the input values. Each node in the hidden layer is connected to the inputs. What is learned in the hidden layer is based on all of the inputs together. In this layer, the network learns interdependencies in the model. Figure 4 shows what happens inside a hidden node.

A simplified explanation of Figure 4 is that a weighted sum is performed within the node: X1 times W1 plus X2 times W2 through X5 and W5. Furthermore, for each hidden node and each output node, a weighted sum is performed. This represents how interactions occur in the network.

Although a closed mathematical theory for linear time-invariant systems exists, nonlinear systems lack an overall theory. To combat this, sometimes nonlinear systems are linearized around their operating points. Subsequently linear methods applied. The design of "universal" modules and structures for nonlinear systems, which can be used for identification, prediction, and control, remains an important issue. However, ANNs can learn nonlinear relationships relatively easily if sufficiently measured data and computing power are available. The learning ability of ANNs may help overcome the difficult mathematical analysis required to solve system identification and control problems in complex and highly nonlinear systems. Source: http://www.zsolutions.com/light.htm

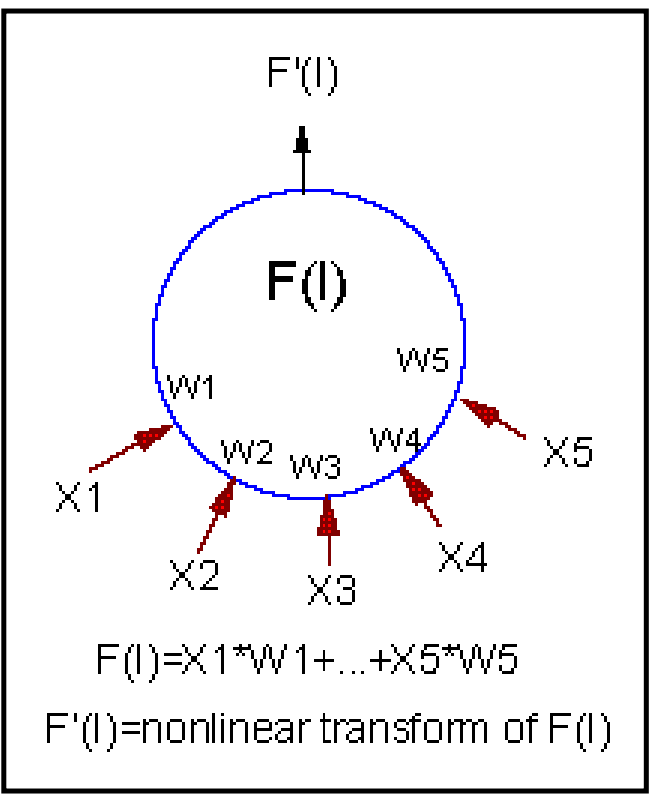

Figure 4. Inside a hidden node.

(http://www.zsolutions.com/light.htm) 


\section{Case Studies Using Advanced Technologies for Operation and Maintenance of Pollution Control Equipment}

\section{Vibration Interpretation Using Simulation and the Intelligence of Networks (VISION) Research Project}

The VISION Project is a collaborative industrial research project under the Brite EuRam III initiative. The Project commenced on 1 May 1996, and was scheduled to run for 3 years. There are nine partners in four countries engaged in the Project, which has a total budget in excess of $3 \mathrm{MECU}$.

The objective of this project was to develop an intelligent, adaptive monitoring and diagnostic system. The system, based on artificial intelligence and simulation modules, analyzes vibration spectra data to sustain high-level equipment reliability. The artificial intelligence derives from the integration of neural networks and knowledge-based systems. The identified aims of VISION are:

- to develop a first level physical model (test rig) to represent a class of simple rotating machines (a rotor suspended between two bearings)

- to develop a finite element model to mimic the vibration signals generated by the test rig in both no defect and defect modes

- to verify the accuracy of the finite element model by comparing its output to actual data from the test rig under controlled experimental conditions and from actual plant data

- to develop an intelligent software module using neural networks and knowledge-based systems to optimize the parameters of the finite element model that will bring its output close to real data generated by the test rig or equivalent simple machines

- to develop a diagnostic system to determine the operating state of the test rig and subsequently the plant of the end-user, from observed real data

- to develop a more complex second level physical model (test rig), by adding couplings and additional rotors and bearings 
- to expand the finite model to imitate the vibration signals generated by the complex test rig

- to compare the output from the expanded finite model to actual data from the second level test rig and actual plant data from the end-user sites

- to expand the intelligent optimization software module to accommodate a greater number of model parameters to represent more complex physical systems.

Source: http://157.228.102.29/vis-info/vis_home.htm

\section{Eli Lilly Study}

The goals of the Eli Lilly study, conducted between February and October 1997, were:

- to find the most efficient approach to identify misalignment problems in flexible coupling systems

- to isolate the source of heat energy in a coupling

- to identify different approaches to problem identification

- to identify problems that are associated with over- and under-tension of belt driven mechanical systems and the implications of over lubrication in bearings

- to quantify over consumption of power in a misalignment system.

The procedure included setting up an apparatus on which various types of flexible couplings were mounted between a 10 horsepower drive motor and a driven shaft that was adjustable to provide controlled misalignment. The study employed a Fixturlaser Shaft 100 laser alignment system with one-micron resolution to control the positioning of the shafts, as precise positioning of the apparatus was a key to the success of the study. Researchers recorded the following observations:

- motor current signature

- motor temperature via thermocouples

- motor and coupling temperature via infrared thermographic imaging

- motor bearing vibration spectra

- coupling airborne ultrasound spectra

- bearing contact ultrasound spectra

- load cell output. 
The study provided the following general conclusions and recommendations:

- In most cases axial vibration exceeded radial vibration. This confirms the rule that misalignment may be a cause when axial vibration is as great as 50 percent of the radial vibration.

- Sometimes misalignment causes high vibration at $2 x \mathrm{rpm}$, indicating that response characteristics depend on coupling design and speed.

- Coupling design impacts the amplitude of the vibration when various misalignment conditions are present.

- Misalignment diagnosed solely from spectral data should be verified using phase data and ancillary technologies.

Further study is warranted to evaluate the effects of bearing condition on results.

Although the results were mixed, the study supplemented the knowledge bank of information on rotating equipment and the application of advanced maintenance technologies to detect problem situations. The study researchers advocate a "large toolbox approach" to determine problems in systems or system components. The "large tool box approach" involves the consideration and possible application of a variety of technologies to detect problems in mechanical and electrical systems.

Subsequent to the study, Eli Lilly suggested the following approach:

1. Find problems or potential problems quickly.

2. Prioritize repairs.

3. Make corrections as needed.

Using infrared as a screening tool and applying the practices mentioned above, Eli Lilly has been able to inspect and rate or prioritize three times the amount of equipment for repair than before instituting this approach (Kelch 1998).

\section{General Electric (GE) Study of Primary Coiling Bearing Run-In Equipment}

The objective of the study that commenced in May 1998 was to design a control system for the primary coiling spindle run-in equipment. The following subsystem was included in the control system: spindle speed display, spindle speed control, bearing temperature monitor/feedback control and a PC user interface with data logging capabilities. To ensure full life from bearings, it is necessary to 
run them in before placing them in service. The run-in system detects manufacturing defects in the bearings. Four sets of bearings support the primary coil, and although the bearings are rated for 18 months of operation, they are failing in 6 months. The reason for the excessive failure rate may be due to an inconsistent run-in process. Subsequently, General Electric plans to use the run-in data to predict failure and to determine the cause of premature failures.

The run-in process operates by varying the shaft speed from 2,000 RPM to 30,000 RPM for a 10-hour period. The three methods for varying the shaft speeds for this time period are:

1. To run the shaft at maximum speed for a time, then stop the shaft and allow the bearings to cool, and then repeat the process

2. To continually increase the shaft speed over the entire time period

3. To increase the speed in steps over the run-in period.

Since the step method appears to cause some stress on bearings, GE researchers ran tests to study step-size and step-length.

To ascertain the minimum step-size that should be used, researchers investigated the dynamic system, based on the foll owing three characteristics:

- The possibility of speed instability at the start of the process

- The effects of vibration at resonant and harmonic frequencies

- Speed surges occasioned by motor defects.

Investigators studied each of the above three characteristics and suggested some guidelines for determining a suitable step-size:

- Instability could occur with small step changes. Because one measures speed by counting the number of revolutions for a fixed time period, less error occurs in measuring speed at higher speeds.

- Vibration effects were investigated. Researchers first established a resonant frequency and plan to make accurate measurements to determine consistent natural frequencies. Factors that affect natural frequencies and that need to be verified include: the bore diameter of the shaft collar, the bearing quality, and accelerometer testing over the range of operation on spindle assemblies.

- Motor wear affects the determination of step-size. Researchers found that if step size is not greater than 1,500 RPM, then surging of the starting torque may affect the settling time of the step. 
The dynamics of the thermal system determine the minimum step-length. $R e-$ searchers used two sets of sensors to conduct testing of the temperature sensors, and $\mathrm{K}$-type thermocouples to verify the temperature sensor readings. Data were collected during the 78-minute test.

Investigators completed initial modeling of the run-in process and ran an 8-hour test to verify the initial modeling of the system. The test data and the initial system model were nearly identical.

The existing run-in system is an open loop system. Consequently, significant transients and variations of speed occur during the run-in process. To remedy this, it was decided to convert the open loop system to a closed loop one. The first step was to analyze and model the open loop system. The method used was to estimate the model from input/output data. Investigators required two parameters from the experiment, the DC gain and the motor time constant. When these data were obtained from the test-run, they were plotted to obtain the steady-state response curve. To determine the transient response of the plant, the steady-state relationship was used to generate three input signals at three operating points. Researchers plotted the data obtained, overlaid with the input signal. Inspection of the graphs showed that the system could be modeled with a first order system. A first order model that provided a good approximation of the plant was produced, using a least squares error modeling technique.

After considering all of the factors associated with the run-in process, the researchers made recommendations as to step-size, step-duration, and number of steps:

- Step-size

- No less than 2000 RPM

- Avoid 16,000 as a step

- Best operating point: 2500-3500 RPM

- Step-duration

- No less than 30 minutes

- Monitor long term bearing temperature around 30,000 RPM

- Best operating point: 30 minutes - 1.5 hours

- Number of steps

- Greater than 5

- Less than 20

- Best operating point: 7-12 steps

- GE researchers believe that the proposed new unit will improve the run-in process for their primary spindles (Dubrawski et al. 1998). 


\section{Reliability Theory}

\section{Reliability Theory Development}

Reliability Theory (RT) was originally developed as a way of describing the statistical performance of equipment using measures like mean time-to-failure (MTTF), hazard rate, or system availability. But as RT became more sophisticated, researchers found that RT excels at describing the effectiveness of maintenance policies.

The field of RT has grown steadily since the 1930s. Reliability Theory's growth has been spurred by increasingly complex applications and increasingly sophisticated statistical methods. Arguably, RT went through its two largest growth spurts when applied to vacuum tube based computers in the 1940s and when applied to semiconductor fabrication plants in the 1990s.

Early computers required thousands of vacuum tubes. By today's standards, they computed very slowly. Without optimized maintenance procedures, the mean time to failure could easily fall below the time required to run a complete program. This forced the electrical engineers responsible for maintenance to think carefully about the consequences of their procedures. The optimal maintenance policies these engineers invented are quite useful for optimizing maintenance strategy in the Army environment.

During the 1980s and 1990s semiconductor fabrication plants rapidly increased the density of devices on a single chip. The machines used to produce such fine patterns on silicon wafers were terribly expensive photolithograph machines. In addition, these photolithograph machines each had a unique set of optical aberrations that could not be reproduced exactly in another machine. Silicon chip designs incorporate several layers of devices that have to appear in exact registration with other devices in layers both above and below. Therefore when adding a new layer, the wafer is brought back to the same photolithograph machine several times. Plants which are required to route a part to the same machine several times are said to be "reentrant."

The scheduling policies that optimize re-entrant plant performance are still poorly understood but several recent advances in queuing theory have yielded 
methods that are superior to commonly used heuristic (exploratory self-taught) methods. The modeling tools that describe re-entrant plants also describe the maintenance process at the Department of Public Works (DPW) level.

The initial models obtained in RT describe the behavior of a set of machines or parts that make up an entire system. For many years it was assumed that RT would advance as these models improved. This assumption was false in several important respects. From a mathematical standpoint, there are several common time-to-failure (TTF) distributions that can easily be justified using sound physics and engineering principles. Unfortunately these functions are sufficiently similar to one another that it takes a large number of experiments or vol uminous field data to determine which function is correct. Appendix A gives an important example of this. Furthermore, there are practical problems with such models. If we own a motor and have a reasonable model describing its expected service life, there is little we can do to influence the situation. We are the motor's owner, not the manufacturer. Therefore, we only have control over our own maintenance policy. This is a common situation and has had a strong influence on RT research.

We will show that the true power of RT lies in the optimization of our own maintenance policies. Also, we will show that modern methods allow us to predict diverse effects of our maintenance policies, including environmental impact, staffing levels, budgets, etc.

The balance of this chapter summarizes the important results of RT. Actual derivations are included in the appendixes to this report for material that is not covered well elsewhere. References are given to other publications that give good expositions.

\section{Application of Reliability Theory}

One might ask, "Do we really care about exact failure mechanisms?" In fact, the answer might well be, "many times, we do not." At first this may seem strange. After all, we are attempting to optimize maintenance strategy with respect to failures of individual components. A simple example illustrates our counterintuitive answer. Suppose we have a device, for example, an electric motor. If we conjecture about the likely failure mechanisms, two are readily apparent. First, the device could fail as a function of how many hours it has been operated in total. (An example of this mechanism is called "wear out.") Second, we could guess that the device might fail as a function of how many times it has been turned on and off. (An example is a failure due to "thermal cycling.") We might 
expect the TTF distribution for each of these processes to be different, and we might believe it to be important to know which process dominates the service life of our device. Surprisingly, the TTF distributions of these two mechanisms are so similar that they have no significant impact on our predictions. Appendix A gives a proof of this.

The similarity of these two TTF distributions is a typical situation, which has several important consequences. First, with little data we can easily come up with a good guess of what the TTF distribution looks like and make reasonable guesses about the predicted service life of the device. In this way, we say that RT methods have "good predictive power." By the same reasoning, we possess little information about how to extend the service life of the device. If thermal cycling dominates the machine's life, it would be better to run it less often and for longer periods. On the other hand, if accumulated run time dominates the machine's service life, it would be better to run the machine more often for shorter periods. Because the TTF distributions are so similar, we cannot prescribe which course to take. For this reason we say that RT has "poor prescriptive power." Experiment is the best way to find out which failure mechanism dominates.

Problems of this type where there are trade-offs between predictive power and prescriptive power, and where we are trying to guess the TTF distribution in the smallest number of experiments, are called problems of "system identification."

In general, it takes an unacceptably large number of experimental trials to discriminate between two similar TTF distributions. For more detail see Wolstenholme (1999).

\section{Hazard Rate - A Reliability Theory Measure}

Hazard Rate is defined as the fraction of working devices that fail per unit time. We shall show that it is one of the most useful measures in classical RT. A derivation of the following facts can be found in (Wolstenholme 1999).

1. There is a one-to-one mapping between the TTF distribution and the Hazard Rate. That is, each TTF distribution has a unique Hazard Rate and each Hazard Rate has a unique TTF distribution.

2. If the Hazard Rate is either constant or monotonically decreasing, the system it describes is called a "happy system." If the Hazard Rate monotonically increases, the system is called an "unhappy system." 
3. RT can be used to derive an optimal maintenance schedule for all unhappy systems.

4. RT gives no analytical method of optimal maintenance for happy systems.

The reason for facts 3 and 4 is simple. The life expectancy of a happy system is either constant or increases with time, assuming the system is still running. That is, if we observe that a happy system is still running, our estimate of its life expectancy is either as long as a new machine, or even longer. The prime example of a happy system is software. Each time a bug is removed from a piece of software the next bug will either be just as hard to find as the previous one or even harder. If a piece of software runs correctly, the best maintenance policy is to let it continue to run (i.e., "Don't mess with it"). That is, any preventative maintenance we perform on the software is more likely to induce a new bug than fix an, as yet, undiscovered bug. (The reader may notice that this is one of the reasons that the so called $\mathrm{Y} 2 \mathrm{~K}$ or millennium bug is such a big problem. Many pieces of old software are so well debugged, that companies use them until they are far beyond obsolescence.)

It should be clear why classical RT cannot yield an optimal maintenance strategy for happy systems. If you observe that your happy system is running, the best strategy is to let it keep running!

An interesting sidelight is to notice that the dividing line between happy and unhappy systems is when the hazard rate is constant. This situation means that the TTF distribution is exponential. This explains, in part, why the exponential distribution is so useful in RT.

However, the existence of happy systems poses a puzzle. If we need a large sample of systems to determine if our system is happy or unhappy, how do we say anything meaningful about optimal maintenance in the mean time? Arriving at a meaningful answer to this question represents the dividing line between classical and modern RT.

\section{Can We Cope with Happy Systems?}

Should we abandon RT when we do not know if the system is happy? No. Can we say anything meaningful about optimal maintenance of happy systems or about systems in general? Yes. The quickest way to see this is to study the mathematical definition of system availability. If we compute the fraction of time that the system is available for work it is easy to show that: 
where:

MTTF = mean-time-to-failure

MTTR = mean-time-to-repair

That is, the system's availability depends on the MTTF which, in many cases, we cannot influence much because it was implicitly set by the equipment manufacturer. But the systems' availability also depends on the MTTR over which we have complete control.

This is the observation that makes RT useful in all situations, and has the largest impact on how optimal maintenance systems are structured. In fact, many modern researchers believe that this observation is so important that they refer to the resulting maintenance systems as implementing RCM. But drawing a sharp chronological dividing line between RT and RCM is misleading. It took many years for researchers in RT to realize that this scenario of reducing MTTR arose repeatedly.

\section{Non-Destructive Evaluation and Happy Systems}

Can a non-destructive evaluation (NDE) work with happy systems? Yes. Recall the definition of a happy system. When conditioned on the observation that the system is still running, a system whose life expectancy remains constant or increases is called a happy system. However, certain methods of NDE may exist that still accurately predict the demise of the system. That is, conditioned on the NDE observation, the system's life expectancy can decrease. To those unfamiliar with probability, this can be confusing at first. The easiest way to explain is that the NDE method may yield new information beyond the simple observation that "the system still runs." NDE can give early warning of system failure and allow the system operator to initiate procurement of appropriate components before they fail. In this way, the MTTR can be reduced thus increasing system availability.

The CERL-engineered management system for pavements has been in use at various municipalities and installations for many years. The PAVER system (Ginsberg, Shahin, and Walther 1990) has many components, but this exposition focuses on only two: 
1. There is an adaptive algorithm that predicts pavement degradation as a function of time. The adaptation has two purposes. First, it can adapt to local conditions such as pavement constituents, weather, and usage. Second, this adaptation implicitly gives rise to a modified version of Hazard Rate (for specialists, we are re ferring to the rate of change in the pavement condition index $[\mathrm{PCl}]$. I nitially, it would seem that, if PAVER detects a constant or decreasing hazard rate (constant or decreasing rate of $\mathrm{PCl}$ degradation), that the system should recommend that the user discontinue usage of PAVER.

2. A second component of PAVER allows users to predict their maintenance needs over an entire road network, and as a side effect, show them how to plan their out-year budgeting with minimal variation. This allows maintenance policy to harmonize well with budget policy. By correctly predicting the amount of money per year needed to maintain a particular road network and by minimizing the year-to-year variation in road repair dollars, PAVER would still be considered a useful tool even in situations where it does not reduce the overall cost of pavement maintenance.

\section{Reliability Centered Maintenance}

RCM recognizes the value of an organization's personnel and takes advantage of their extensive experience running the facility/equipment. The following categories can be used to assist in classifying maintenance of equipment:

- Corrective Maintenance (CM) or "run-to-failure" works on the assumption that it is most cost-effective to al low equipment to run unattended until it fails. Corrective Maintenance is used on the lowest priority equipment.

- Preventive Maintenance (PM) is based on performing maintenance tasks on equipment at regular intervals, regardless of whether maintenance is actually needed at the time.

- Predictive Maintenance (PDM) is based on real-time data collected from a piece of equipment. These data show the current status of the equipment.

- Proactive Maintenance (PAM) determines the root causes of failure. This involves going to the manufacturer for equipment redesign to avoid future breakdowns of the equipment (Reliability Centered Maintenance [RCM ] Tutorial and Application).

\section{Harmonizing Reliability Centered Maintenance with Army Policy}

RCM can, on occasion, reconcile disparate views of maintenance policy. In the early 1990s, the Army decided on the basis of impending reduction in funding 
that the infrastructure part of the budget would be cut rather than the training budget. The decision was made that the policy of run-to-failure would be the most cost-effective means of equipment maintenance. In the Army's accounting system, maintenance dollars come from a fixed funding line item. It makes no sense to organize an optimal maintenance program, because the cost of inspection and early replacement would only deplete maintenance funds. But in the event of equipment breakage, the Army accounting system produces a minor miracle. The cost of repairing broken equipment on an emergency basis comes from the "capital improvements" budget that is separate from the maintenance budget. By using a strict run-to-failure policy, equipment managers can maximize the funds used on their equipment.

Unfortunately, it has been known for many years that this run-to-failure policy is the single fastest way to reduce the MTTF and decrease system availability. An example of this concept is the maintenance of vacuum tube computers. We will outline the methods here using this example because it contains several instructive simplifications that will become apparent shortly.

Consider a computer made up of a large number of vacuum tubes, each of which has the MTTF distribution shown in Figure 5. If we begin using a new computer containing new vacuum tubes and replace tubes as they fail during the first wear out cycle, the number of tubes replaced per unit time will look like the first hump in Figure 6.

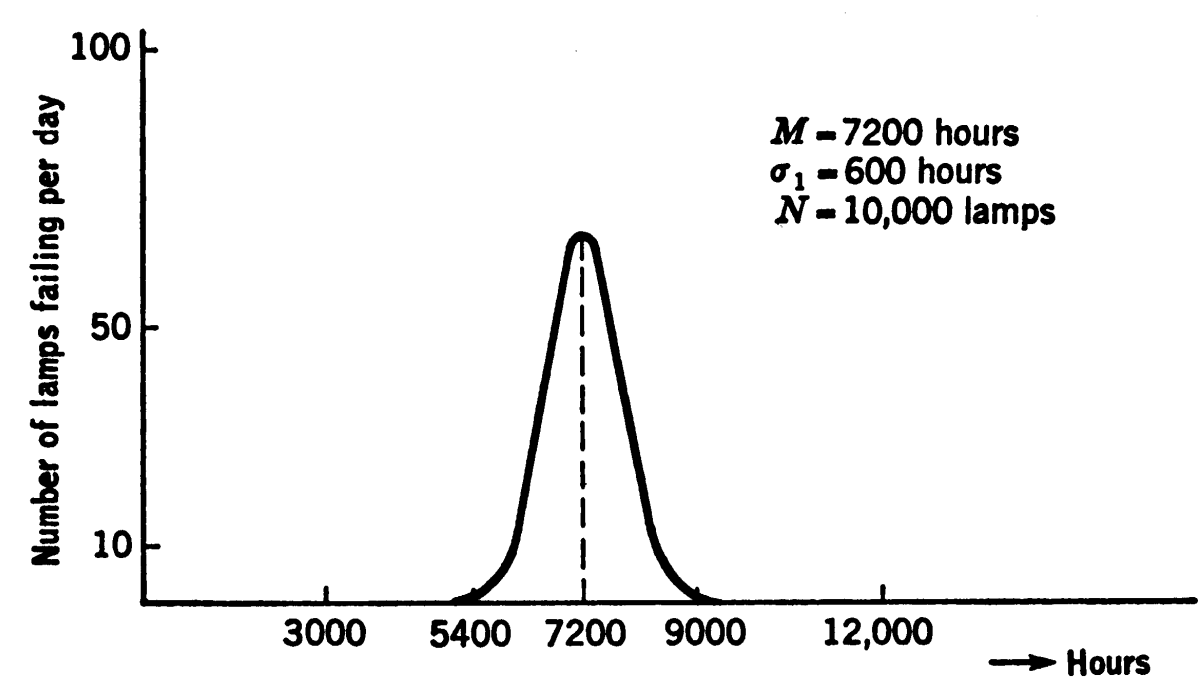

Distribution of lamp life.

Figure 5. Distribution of lamp life (Bazovsky 1961). 


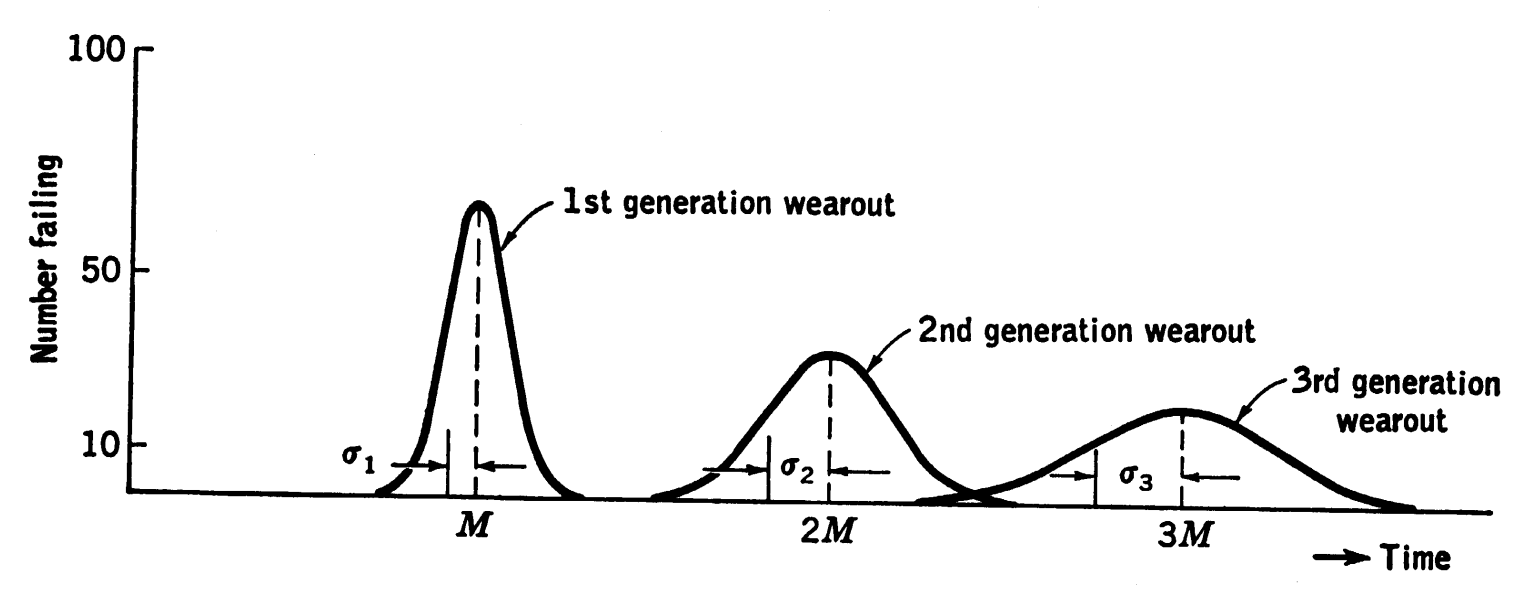

Wearout curves of three lamp generations.

Figure 6. Wearout curves of three lamp generations (Bazovsky 1961).

Notice that we enjoy a long period of reliable operation until the tubes begin to wear out. In the second wear out cycle, the number of tubes replaced per unit time will look like the second hump of the graph in Figure 6. The third wear out cycle will look like hump 3 in Figure 6 and so forth. (Note for specialists: The shape of hump " $\mathrm{n}$ " is the convolution of hump n-1 with hump 1.) The number of tubes replaced per unit time will be the sum of all of these humps and will look like the upper line of Figure 7. For further details, see Bazovsky (1961).

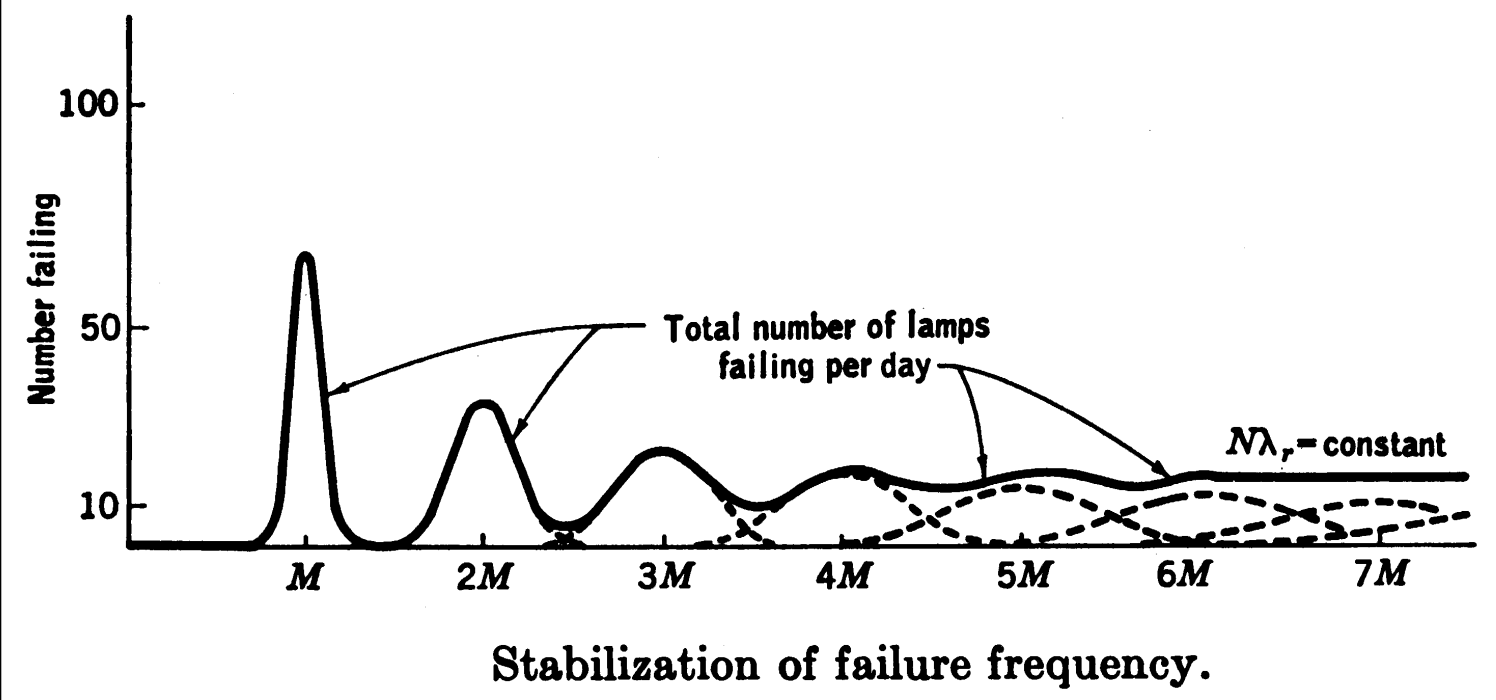

Figure 7. Stabilization of failure frequency (Bazovsky 1961). 
As time goes on, we will be replacing tubes constantly, this means that we have driven the MTTF down as far as it can go, and have exceptionally low availability.

An alternative explanation is as follows. This is the reason people buy new cars! The idea behind buying a new car is that all of the parts of the car are new at the same time. This is analogous to the large time between when we begin running the computer and the first wear out cycle in Figure 6. As a car gets older, its components are replaced as needed so they are widely varying ages, and it seems like something is always going wrong. This is analogous to the high time behavior of Figure 7.

Thus we see that a run-to-failure policy is terrible for long term availability. Is there an alternative way to interpret Army policy, maximize our maintenance budget, and maximize the MTTF? Yes. If we keep track of mean service life of the unhappy components in our system an alternative policy is possible. We wait until breakdown. This lets us tap into the capital improvements budget. We then use these funds to fix the broken component and replace all other components that are beyond, or near their expected service life. This is sometimes called "renewing maintenance" in that it tries to achieve the longest possible period of high reliability between breakdowns. In this way we try to repeat the high reliability period before the first wear out peak of Figure 6, again and again. In the era of the vacuum tube based computers, engineers settled on the following maintenance procedure. Run the machine until the first vacuum tube blows, then replace all tubes in the blown tube's sub-system. This strategy was found to yield acceptable MTTF. 


\section{Queuing Theory}

A recent approach to RCM has come from new methods of queuing analysis. Queuing theory addresses the analysis of systems where many jobs are waiting for some service. Appendix B includes a summary of our current understanding of the behavior of closed queuing networks. It is important to remember that this is currently an active area of research. The contents of Appendix B are current, but will be quickly outdated over the next year.

Queuing theory analysis can yield important information about the efficacy of maintenance procedures. From a queuing perspective, maintenance procedures are merely networks of jobs that await service from maintenance personnel. Although queuing theory has been used in one form or another since the early 1900s, recent advances allow the analysis of much more complex networks. In the context of RCM, the most important developments of queuing theory can cope with several complexities common to most maintenance shops.

\section{Re-Entrant Lines}

In maintenance shops, the same job may require service from a single person several different times. For example, personnel responsible for procurement may see a single job several different times in the procurement cycle.

\section{Multiple Job Classes}

Maintenance personnel have many different types of jobs waiting for them at any one moment. They do not choose the next job randomly. They can distinguish one job class from another and make a decision consciously.

\section{Service Policy}

At any one moment, one maintenance worker may have several different jobs of different classes waiting for service. The prioritization the worker assigns to these waiting jobs is called the service policy. 


\section{Stochastic Routing}

In many networks, jobs can be split up among maintenance personnel.

\section{Pre-emptive Scheduling}

If an especially important job arrives for service from a particular worker or set of workers, all other work may be suspended in order to service the important task.

\section{Down Time}

Maintenance workers, machines, or tools can have varying availability based on their own TTF and time-to-repair (TTR) statistics.

\section{Large Networks}

This new style of analysis can cope with enormous and complex queuing networks. The technique was designed to cope with reentrant routing in semiconductor fabrication plants where many products are in process simultaneously. For specialists requiring a detailed introduction to this style of analysis, we recommend the article "Closed queuing networks in heavy traffic: Fluid limits and efficiency" (Kumar and Kumar 1996). 


\section{Queuing Theory Modeling}

One of the objectives of the project was to investigate the application of queuing theory modeling as an operations and maintenance tool for pollution control equipment. A queuing model usually includes one or more servers that render the service, a pool of customers, and some description of the arrival and service processes. If there is more than one queue for a server, then there may also be some policy regarding which customer receives service. In working with queuing systems, it is easiest to analyze the system in steady state (after the system has started up and things have settled down). Our analysis allows us to optimize for one or more of these performance measures.

\section{Throughput}

How fast do jobs go through the maintenance system? Can one predict how long a particular job will take? Recent analysis of semiconductor fabrication plants indicates that these new methods of analysis were able to speed up fabrication by 30 percent and decrease the variability of completion times by 60 percent.

\section{Queue Length}

How many jobs are likely to be waiting for any one maintenance worker at a given moment? Can a job be expedited without throwing off the schedule of other jobs?

\section{Failure and Repair Statistics}

Recent results show how to predict the equilibrium points of queuing networks (Ginsberg and Kumar 1997). This allows prediction of TTF and TTR statistics for the entire network and, hence, the reliability. 


\section{Staff Levels}

By calculating "what if" scenarios with software, the number of maintenance workers and responsibilities can be optimized.

\section{Budgets}

Are budgets made more predictable? Yes. This is not an obvious result. Because the variability of the output of the system is reduced (see "Throughput" above) the out-year budget planning is more predictable.

\section{Ecological Impact of RCM}

Since "total time to repair" (TTR) statistics can be calculated for all machines, systems and subsystems, other performance statistics can be deduced easily. Of particular interest to the Army are the TTR statistics for pollution control equipment. The environmental impact of Army operations can be quantified as the average cost incurred given the reliability of the system. In this way the maintenance personnel may be able to justify increased expenditures, internal priority changes, and improved procurement documents because they can predict the total cost to the Army in advance. 


\section{Conclusion}

This study reviewed innovative methods of performing maintenance of pollution control equipment, specifically for application at Army installations. Previous CERL work has shown that RCM usually depends on test regimens rather than approaching the subject from a statistical viewpoint even though statistics have been used in manufacturing quite successfully. Statistical maintenance modeling, even when approached from different initial viewpoints, reveals the impact of different maintenance policies. Large scale systems have escaped effective analysis until recently. This study has summarized recent attempts to model large systems by using a model based on queuing theory. Queuing theory makes statistical predictions that summarize how long it takes to repair a broken system, and predicts the availability of the system.

By studying the methods being used now for RCM and the military's predilection to run-to-failure maintenance, this research has produced a linear program from the queuing model that can help reduce the downtime of the equipment, which is the intent of RCM.

This study concludes that using these recent methods may allow U.S. Army installations to optimize maintenance policy for minimal ecological impact. 


\section{References}

A Manager's Guide to Neural Networks:

http://www.zsolutions.com/amanager.htm

Approximate Realizations and Control of Dynamical Systems via Artificial Neural Networks: http://rodin.wustl.edu/doc2.html

Artificial Neural Network (ANN) and Condition Monitoring: http://www.zsolutions.com/light.htm

Basic Theory: http://amath.colorado.edu/appm/faculty/jdm/faq-[2].html

Bazovsky, I gor, Reliability Theory and Practice(Prentice-Hall, 1961).

Beltrami, Edward, Mathematics for Dynamic Modeling, 2d ed. (SUNY, Stony Brook, NY, 1997): http://www.europe.apnet.com/textbook/pecs/new9798/beltrami.htm

Chalifoux, Alan, J earldine I. Northrup, and J oyce Baird, Re iability Centered Maintenance (RCM): Operating a MoreEffective Maintenance Program, Technical Report (TR) 99/41/ADA367999 (U.S. Army Construction Engineering Research Laboratory [CERL], April 1999).

Chalifoux, Alan, J earldine I. Northrup, and Nina Y. Chan, Reliability Centered Maintenance (RCM): Tutorial and Application (RCM.ppt/ R6.exe), TR 99/50 (CERL, May 1999).

College of Engineering, I ndustrial Technol ogy I nstitute and General Motors (Research collaborators include O. Tekinalp, Diana Rincon, J . MacBain, K. Harding, D. Stephenson, and J . Agapiou), "Dynamic Modeling and Analysis of Drill Bit Vibrations": http://www-personal.engin.umich.edu/ ulsoy/Drills.html

Comparison of Continuous and Discrete Models: http://www.sysc.pdx.edu/F aculty/Wakeland/529cvsd.htm

Dai, J .G. "On Positive Harris Recurrence of Multiclass Queuing Networks: A Unified Approach Via Fluid Limit Models," Annals of Applied Probability, vol 5 (1995), pp 49-77.

"Distribution Failure Prediction System" (Kevin System Co., Ltd.): http://www.keyin.co.kr/eng/Product/1/d_ 7.html

Dubrawski, Richard, J ohn Hines, J im O'Malley, J eff Payne, Dave Peters, and Michael Sheley, General Electric - Primary Coiling Bearing Run-In Equipment (4 May 1998). 
"Dynamad - Dynamic M odeling of Mechanical Systems," Dynamad SARL, Z.I de la Chatelaine, Rue Rene Cassin, Gaillard, 74240 France: http://software-guide.com/cdprod1/swhrec/005/850.shtml

"Dynamic Modeling," Mechatronics: http://mechatronics.me.vt.edu/book/section3/dynamicmodel.html

"EASY5 Modeling, Analysis, and Simulation" (Boeing Company): http://www.boeing.com/assocproducts/easy5/easy5 intro.html

Failure Analysis (Bass Associates Inc.): http://www.bassengineering.com/failana.htm

Failure Mode and Effect Analysis (FMEA) (Bass Associates Inc.): http://www.bassengineering.com/FMEA.htm

Fault Tree Analysis (FTA): http://www.bassengineering.com/FTA.htm

Fluorescent Penetrant Replica Test (FPRT): http://janus.nwra.com/nwra/PenScan.html

Ginsberg, Mark, M.Y. Shahin, and J .A. Walther, AutoPaver, A Software Packagefor Automated Pavement Evaluation, TR M-90/15/ADA224176 (CERL, A pril 1990).

Ginsberg, Mark, and P.R. Kumar, "Throughput of Closed Queuing Networks with Multiple Loops," Transactions of the CDC (1997).

Hannon, Bruce, and Ruth Matthias, Dynamic Modeling (Springer-Verlag, New York, 1994): http://software2.bu.edu/CEES/toc_ruth.html

Harrison, J .M., and V. N guyen, "Some Badly Behaved Closed Queuing Networks," in Frank P. Kelly and Ruth Williams, eds., Stochastic Networks, vol 71, pp 117-124 (Springer-Verlag, New York, 1958).

Harrison, J .M., and L.M. Wein, "Scheduling Networks of Queues: Heavy Traffic Analysis of a TwoStation Closed Network," Operations Research, vol 38 No. 6 (1990), pp 1052-1064.

Infrared Thermography: http://www.ozemail.com.au/-its3d/thermog.html

J eong, Kyung-Hun, and Yosinori Iizuka, "Effective Prediction of Failure Modes Based on the Concept of 'Association' and 'Hierarchy,"' Quality, vol 26, No. 4 (1996), pp 91-99: http://www.tam.t.u-tokyo.ac.jp/-kyunghun/paper2.html

J eong, Kyung-Hun, and Yosinori I izuka, "Effective Cause \& Effect Analysis of F ailure Modes Using the Failure Mode Mechanism Diagram," Quality, vol 27, No. 4 (1997), pp 108-116: http://www.tam.t.u-tokyo.ac.jp/-kyunghun/paper4.html 
J eong, Kyung-Hun, H. Kume, and Y. I Izuka, "Implementing FMEA through Multiple Points of View," Proc. $9^{\text {th }}$ AQMS (1995), pp 85-90:

http://www.tam.t.u-tokyo.ac.jp/-kyunghun/paper1.html

J in, H., J . Ou, and P.R. Kumar, "The Throughput of Closed Queuing Networks: Functional Bounds, Asymptotic Loss, Efficiency, and the Harrison-Wein Conjectures," to appear in Mathematics of Operations Research.

Kelch, Craig, “Cross Technology Study at Eli Lilly," Maintenance Technol ogy (September 1998), pp 15-20.

Kumar, P.R., and T.I. Seidman, "Dynamic Instabilities and Stabilization Methods in Distributed Real-Time Scheduling of Manufacturing Systems," IEEE Transactions on Automatic Control, vol AC-35, No. 3 (March 1990), pp 289-298.

Kumar, Sunil, and P.R. Kumar, "Performance Bounds for Queuing N etworks and Scheduling Policies," IEE E Transactions on Automatic Control, vol 39, No. 8 (August 1994), pp 1600-1611.

Kumar, Sunil, and P.R. Kumar, "Closed Queuing Networks in Heavy Traffic: Fluid Limits and Efficiency," Stochastic Networks: Stability and Rare Events, Lecture Notes in Statistics, P.

Glasserman, K. Sigman, and D. Yao, eds., vol 117 (Springer-Verlag, New York, 1996), pp 41-64.

Kunt, Tekin A., Thomas J . M cAvoy, Richard E. Cavicchi, and Steve Semancik, "Dynamic Modeling and Optimization of Micro-H otplate Chemical Gas Sensors":

http://www.engr.umd.edu/ atkunt/research/adchem97/adchem.html

Light Details of Neural Networks:

http://www.zsolutions.com/light.htm

Lu, S.H., and P.R. Kumar, "Distributed Scheduling Based on Due Dates and Buffer Priorities," IEEE Transactions on Automatic Control, vol AC-36, No. 12 (December 1991), pp 1406-1416.

Morrison, J ames R., and P.R. Kumar, "On the Guaranteed Throughput and Efficiency of Closed ReEntrant Lines," submitted to Queuing Systems: Theory and Applications (December 1996).

National Aeronautics and Space Administration (NASA), Re iability Centered Maintenance (RCM) Guide for Facilities and Collateral Equipment (December 1996).

Northrup, J earldine I., Aaron J . Averbuch, J oyce C. Baird, Mark D. Ginsberg, J ames H. J ohnson, Hannon T. Maase, and Veda D. Scarpetta, Guidel ines for Pollution Control Equi pment Components, TR 98/122/ADA361834 (CERL, September 1998).

Object-Oriented Cognitive Decision Support Engine:

http://www.nasec.ctc.com/manuknow/technica.htm

Power Loss of High Speed Spur Gears: http://www.mel.go.jp/mainlab/kiso/kis02e.html

Predictive Diagnosis for Rolling Bearings: http://www.mel.go.jp/mainlab/kiso/kis01e.html 
Rotating Machinery Simulator:

http://altasol.com/rms01.htm

Rybko, A.N., and A.L. Stolyar, "On the E rgodicity of Stochastic Processes Describing Open Queuing Networks," Problemy Peredachi Informatsii, vol 28 (1991), pp 2-26.

Shearer, J ., Lowen Bohdan, T. Kulakowski, and J ohn Gardner, Dynamic Modeling and Control of Engineering Systems, 2e (Prentice Hall Engineering, Science \& Math, 1997): http://www.phptr.com/ptrbooks/esm 0133564037.html

So You Want to Try Neural Networks?: http://www.zsolutions.com/soyou.htm

Statistics Details, Weaver Engineering: http://www.weavereng.com/Statistics_Detail.htm

“STELLA," High Performance Systems, Inc., 1-800-332-1202: http://www.hps-inc.com/products/STELLA/stella.html

Technical Approach for the Manufacturing Knowledge Project: http://www.nasec.ctc.com/manuknow/technica.htm

Tracor - Equipment Monitoring: http://www.aard.tracor.com/Milner/fil.htm

Vibration Interpretation using Simulation and the Intelligence of Networks (VISION) Research Project:

http://157.228.102.29/vis-info/vis home.htm

Weibull Analysis: http://www.bassengineering.com/weibull.htm

Wolstenholme, Linda C., Reliability Modeling: A Statistical Approach (Chapman \& Hall, 1999). 


\section{Appendix A: Time-to-Failure Distributions}

Equipment that fails randomly over its accumulated run time has an exponential time-to-failure distribution. This distribution is:

$$
f(t)=\lambda e^{-\lambda t} \text {. }
$$

The quickest way to see that this is correct is to notice that:

$$
\int_{-\infty}^{\infty} f(t) d t=\int_{0}^{\infty} \lambda e^{-\lambda t} d t=1 .
$$

(i.e., $f(t)$ is an actual distribution), and that:

$$
H(t)=\frac{f(t)}{1-F(t)}=\lambda .
$$

i.e., the exponential distribution is unique in having a constant hazard rate. The probability of failure on or before time $t$ is:

$$
\int_{0}^{t} \lambda e^{-\lambda t} d t=1-e^{-\lambda t} .
$$

This is an important result, as will be shown shortly.

Now consider a system that fails due to on-off cycles, for example, via thermal cycling. Suppose the probability that the system fails in any one cycle is " $p$," then the probability that it fails on cyclen is:

$$
p(n)=(1-p)^{n-1} p .
$$

That is, the system ran n-1 times and failed on the n-eth trial. This is a Bernoulli distribution with parameter $p$.

There are several ways to observe that the Bernoulli distribution is closely related to the exponential distribution. 
First, consider what we might mean by "a trial" in the Bernoulli distribution. For an electric motor that undergoes thermal cycling each time power is applied, this is obvious. Each time power is applied to the motor, we call this a trial. However, the distinction is not always as clear. Suppose we are meteorologists, and want to talk about quantities like a "30-year rain." At first, we may consider that this means we are talking about a Bernoulli distribution with $p=1 / 30 y e a r s$ and $\mathrm{n}=$ number of years. I $\mathrm{n}$ this way the chance of observing such a rain within $n$ years is:

$$
\sum_{n=0}^{N}(1-p)^{n-1} p=1-(1-p)^{n+1} .
$$

This result looks reasonable at first, but on closer inspection, this answer has several features that are difficult to explain. If $\mathrm{n}=$ number of years, this answer makes no sense from the standpoint of dimensional analysis where the exponent should be dimensionless. If there is more than one rain per year, then the number of trials should not be in units of years, but in units of rainstorms. This is easily fixed by introducing a constant alpha = rains per year, and the above re sult becomes:

$$
1-\left(1-\frac{p}{\alpha}\right)^{\alpha n+1} \text {. }
$$

This lays bare the real objection. This answer should be invariant by choice of alpha (but is not). The time units in which we measure the result should not change the answer. Luckily we can take a limit in alpha:

$$
\lim _{\alpha \rightarrow \infty}\left[1-\left(1-\frac{p}{\alpha}\right)^{\alpha n+1}\right]=1-e^{-p n},
$$

which is clearly the exponential distribution associating $\mathrm{p}$ with lambda and $\mathrm{n}$ with $\mathrm{t}$.

Another way is to cal culate the discrete version of hazard rate:

$$
H(n)=\frac{p(n)}{1-F(n)}=\frac{(1-p)^{n-1} p}{1-\sum_{n=0}^{n}(1-p)^{n-1} p}=\frac{(1-p)^{n-1} p}{1-1+(1-p)^{n+1}}=\frac{p}{(1-p)^{2}},
$$

which shows that the discrete hazard rate is constant.

Finally, note that the graphs of these two functions are quite similar (Figure A1). 


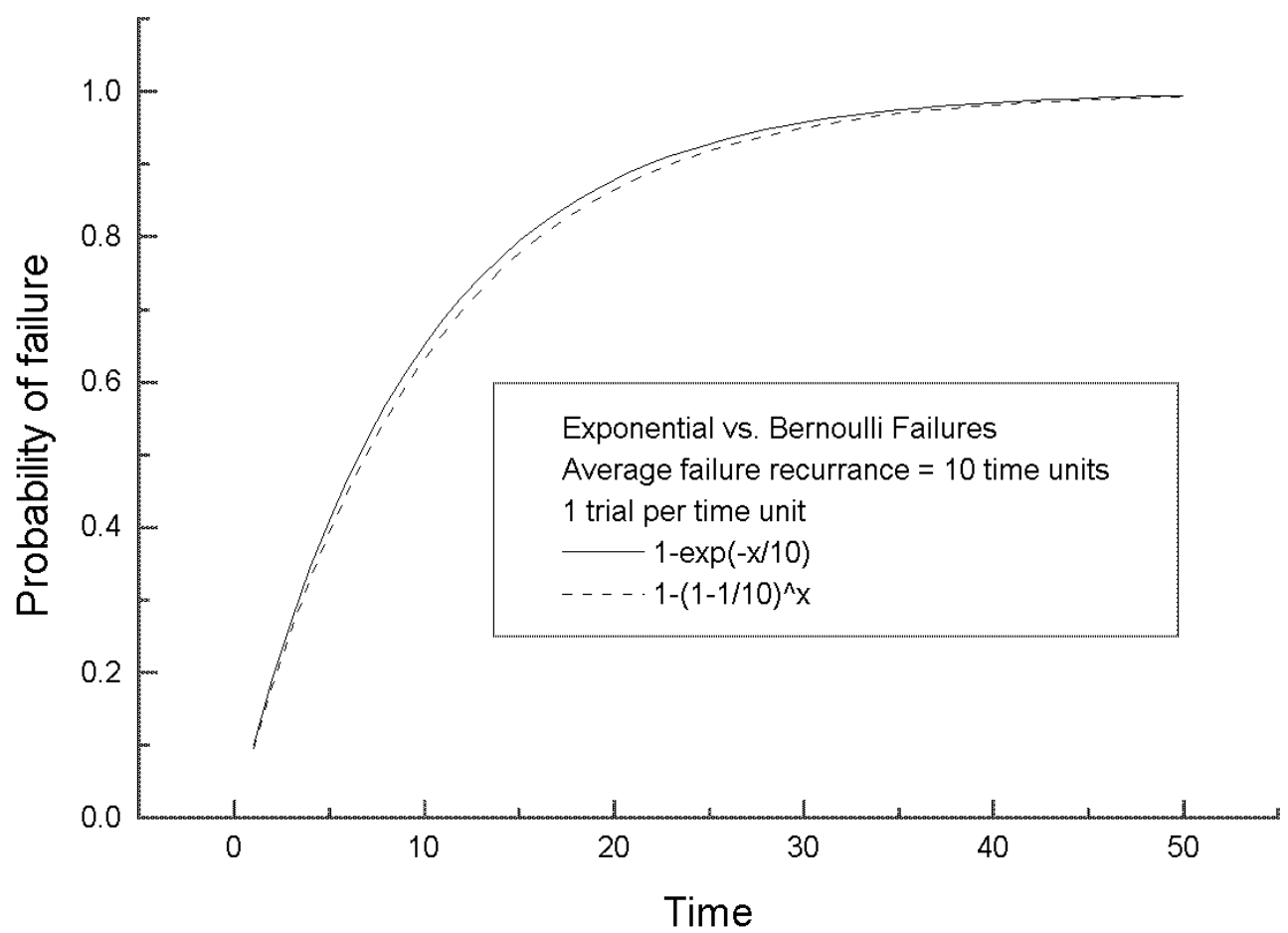

Figure A1. Exponential vs. Bernoulli failures. 


\section{Appendix B: Closed Queuing Networks}

Recently much progress has been made on the study of closed queuing networks with a single route, i.e., when the routing matrix is irreducible. Let $T^{\theta}(N)$ denote the throughput of such a network when the population size is $N$, and the scheduling policy $u$ is employed. For Markovian queuing networks with a single route, J in, Ou, and Kumar were able to establish upper and lower bounds of the form:

$$
\frac{N}{N+\underline{v}} T^{*} \geq T^{\theta}(N) \geq \frac{N}{N+\bar{v}} \underline{T}
$$

for all $N$.

Above, $T^{*}$ is the throughput capacity of the system. Clearly $\underline{v}$ is a lower bound on the asymptotic loss which is defined as:

$$
\lim _{N \rightarrow \infty} \frac{N\left[T^{*}-T^{\theta}(N)\right]}{T^{*}} .
$$

When the quantity $\underline{T}$ in the lower bound is also equal to $T^{*}$ then it follows that:

$$
\lim _{N \rightarrow \infty} T^{\theta}(N)=T^{*}
$$

and the network operated under the scheduling policy is said to be efficient. Moreover, in this circumstance, $\bar{v}$ is an upper bound on the asymptotic loss. In $\mathrm{J}$ in, Ou, and Kumar linear programs are provided for determining the quantities $\underline{\mathrm{v}}, \bar{v}, \underline{\mathrm{T}}$, and $\mathrm{T}^{*}$. The advantage of having throughput bounds as above is that they are functional bounds, i.e., they are valid for all N. Hence the determination of $\underline{v}, \bar{v}, \underline{T}$, and $T^{*}$ serves to bound the performance curve of the network under the given scheduling policy.

In Kumar and Kumar a different approach was taken. There the fluid model approach pioneered by Rybko and Stolyar (1991) and Dai (1995) was extended to the study of closed networks. It was established that the entire class of Last Buffer First Serve (LBFS) scheduling policies is efficient for the case of a 
deterministic closed route, i.e., closed reentrant lines. These are scheduling policies based on buffer priorities, where some arbitrary buffer is designated as the "last" buffer, and priority is given to buffers that are closer to the end. Thus the stability of LBFS for open systems was extended to closed systems.

Earlier, using a formal approach based on Brownian networks, Harrison and Wein (1990) had conducted an analysis of closed networks with two stations, and conjectured that a specific policy, called the HW-policy, was asymptotically optimal. By this is meant that its asymptotic loss is less than that of all other policies. This was done by examining a particular RBM process, and conjecturing a formula for the asymptotic loss of all buffer priority policies. The particular HW policy was characterized by certain indices for buffers, called the HW-indices, which are used to prioritize the buffers. In Kumar and Kumar (1994) it was also proved that the HW policy is indeed efficient for all two station closed re-entrant lines.

The HW policy and the conjectured asymptotic loss formula were examined in $\mathrm{J}$ in, Ou, and Kumar. There it was established that its value for $I$ was indeed equal to $T^{*}$, thus establishing its efficiency. Moreover, it was established that no policy could have an asymptotic loss strictly smaller than the conjectured loss of the HW policy. Simultaneously, an additional condition was identified in Jin, Ou, and Kumar, which is missing in the work of Harrison and Wein (1990) and it was established that under this condition, all non-idling policies are indeed efficient, and moreover no such policy could have an asymptotic loss strictly greater than that of the exact opposite of the HW policy, dubbed there as the Anti-HW policy.

Earlier, it was already established in Harrison and Nguyen (1958) that the closed version of the system in Kumar and Seidman (1990) and Lu and Kumar (1991) was indeed inefficient, thus establishing that not all policies can have finite asymptotic loss, and thus also that the conjectured formula for asymptotic loss in Harrison and Wein (1990) cannot hold in full generality.

More recently, Morrison and Kumar (1996) have turned to examining the issue of necessary conditions for efficiency of all non-idling scheduling policies in closed re-entrant lines. They have established that the condition earlier identified by $\mathrm{J}$ in, Ou, and Kumar as being sufficient for efficiency is actually necessary too, when the inequality is allowed to be non-strict.

All these studies however, have been confined to closed networks with just one loop. In many application areas however, several closed loops may simultaneously exist. One example is communication networks where several origin- 
destination pairs are each controlled by window based flow control policies. A widespread example of this is TCP/IP, which regulates the window size dynamically so as not to overload the network. Another example is manufacturing systems where several part types are made. If the number of parts of each type is regulated at a fixed level, then again one obtains closed networks with multiple routes.

The purpose of the present paper is the study of such closed systems with multiple routes. In such systems there is a population vector made of one population level for each route. Also one has a vector throughput where each component is the throughput of a particular route. Our goal is to study the behavior of the vector throughput as a function of the vector population, both for particular buffer priority policies as well as the class of all non-idling policies.

Consider a network with $B$ stations labeled $b_{1} \ldots b_{B}$. There are $L$ routes (or loops) through the system. The routes are specified by a routing matrix $p_{i j}$, which is the probability of moving next to buffer $j$, after having visited buffer $i$. Buffer $i$ is served by station $\sigma(i)$. Customers in buffer $b_{i}$ require an exponentially distributed service time with mean $1 / \mu_{i}$.

Let $T^{\theta, r}(N)$ denote the throughput of route $r$ under a given scheduling policy $\mathrm{u}$ when the population vector is $\mathrm{N}$. Here $\mathrm{N}=\left(\mathrm{N}_{1}, \ldots, \mathrm{N}_{\mathrm{L}}\right)$ where $\mathrm{N}_{\mathrm{L}}$ is the population of loop route $\mathrm{L}$. Also let $\mathrm{v}=\left(\mathrm{V}_{1}, \ldots, \mathrm{V}_{\mathrm{L}}\right)$ denote the asymptotic loss, when the population proportions in the routes are held constant, but the total population increases to infinity. The main results are the following.

Theorem 1: Bound on weighted throughput.

Let $\alpha^{L}$ be the fraction or the total population stored in loop $L$ of a closed loop system. Let $\pi$ be the concatenation of the steady-state probability vectors for communicating classes of the routing matrix P, i.e., of the various loops so that $\sum_{i \in L} \pi_{i}=1$. Consider first the following linear program with decision variables $\left\{\mathrm{T}^{\llcorner}, \mathrm{q}_{\mathrm{ij}}, \mathrm{w}_{\mathrm{ni}}, \mathrm{u}_{\mathrm{j}}, \mathrm{f}_{\mathrm{i}}^{\llcorner}\right\}$

$$
\max \left(\sum_{L} \alpha^{L} T^{L}\right)
$$

subject to: 


$$
\begin{aligned}
& \underset{\substack{i, j \\
\sigma(i)=\sigma(j) \\
p(i) \leq p(j)}}{\forall} \quad u_{j} \geq \mu_{i}\left(-q_{j, i}+q_{j, i+1}-\frac{f_{i}^{L(j)}}{\pi_{i}}\right) \\
& \underset{\substack{i, j \\
\sigma(i) \neq \sigma(j)}}{\forall} w_{\sigma(i), j} \geq \mu_{i}\left(-q_{j, i}+q_{j, i+1}-\frac{f_{i}^{L(j)}}{\pi_{i}}\right) \\
& \forall T^{L(i)}+u_{i}+\sum_{n \neq \sigma(i)} w_{n, i} \leq 0 \\
& \underset{L^{\prime} \in L}{\forall} \sum_{i \in L} \sum_{L} \alpha^{L} f_{i}^{L} \leq c^{L^{\prime}} \\
& \underset{i, j}{\forall} q_{i, j}=q_{j, i} \\
& \underset{L}{\forall} \sum_{i \in L} \pi_{i}\left(q_{i, i}-q_{i, i+1}\right) \leq c^{L} v .
\end{aligned}
$$

Denote by $T^{*}$ the value of this LP. Consider now a second linear program with the same set of decision variables:

$$
\min (v)
$$

subject to:

$$
\begin{aligned}
& \underset{\substack{i, j \\
\sigma(i)=\sigma(j) \\
p(i) \leq p(j)}}{\forall} \quad u_{j} \geq \mu_{i}\left(-q_{j, i}+q_{j, i+1}-\frac{f_{i}^{L(j)}}{\pi_{i}}\right) \\
& \underset{\substack{i, j \\
\sigma(i) \neq \sigma(j)}}{\forall} w_{\sigma(i), j} \geq \mu_{i}\left(-q_{j, i}+q_{j, i+1}-\frac{f_{i}^{L(j)}}{\pi_{i}}\right) \\
& \underset{i}{\forall} T^{L(i)}+u_{i}+\sum_{n \neq \sigma(i)} w_{n, i} \leq 0 \\
& \underset{L^{\prime} \in L}{\forall} \sum_{i \in L} \sum_{L} \alpha^{L} f_{i}^{L} \leq c^{L^{\prime}} \\
& \underset{i, j}{\forall} q_{i, j}=q_{j, i}
\end{aligned}
$$




$$
\begin{aligned}
& \underset{L}{\forall} \sum_{i \in L} \pi_{i}\left(q_{i, i}-q_{i, i+1}\right) \leq c^{L} v \\
& T^{*} \leq \sum_{L} \alpha^{L} T^{L} .
\end{aligned}
$$

Denote the value of this LP by $\underline{\mathrm{v}}$.

Then:

$$
T^{u}(N) \leq \frac{N}{N+\underline{v}} T^{*} .
$$

Theorem 2: Lower functional bound on weighted throughput.

Consider first the linear program:

$$
\max \left(\sum_{L} \alpha^{L} T^{L}\right)
$$

subject to:

$$
\begin{aligned}
& \underset{\substack{i, j \\
\sigma(i)=\sigma(j) \\
p(i) \leq p(j)}}{\forall} \quad u_{j} \geq \mu_{i}\left(-q_{j, i}+q_{j, i+1}-\frac{f_{i}^{L(j)}}{\pi_{i}}\right) \\
& \underset{\substack{i, j \\
\sigma(i) \neq \sigma(j)}}{\forall} w_{\sigma(i), j} \geq \mu_{i}\left(-q_{j, i}+q_{j, i+1}-\frac{f_{i}^{L(j)}}{\pi_{i}}\right) \\
& \forall-T^{L(i)}+u_{i}+\sum_{n \neq \sigma(i)} w_{n, i} \leq 0 \\
& \underset{L^{\prime} \in L}{\forall} \sum_{i \in L^{\prime}} \sum_{L} \alpha^{L} f_{i}^{L} \leq-c^{L^{\prime}} \\
& \underset{i, j}{\forall} q_{i, j}=q_{j, i} \\
& \forall \underset{L}{\forall} \sum_{i \in L} \pi_{i}\left(q_{i, i}-q_{i, i+1}\right) \leq-c^{L} v .
\end{aligned}
$$

Denote by $I$ the value of the LP, and consider the second linear program: 
$\min (v)$

subject to:

$$
\begin{aligned}
& \underset{\substack{i, j \\
\sigma(i)=\sigma(j) \\
p(i) \leq p(j)}}{\forall} \quad u_{j} \geq \mu_{i}\left(-q_{j, i}+q_{j, i+1}-\frac{f_{i}^{L(j)}}{\pi_{i}}\right) \\
& \underset{\substack{i, j \\
\sigma(i) \neq \sigma(j)}}{\forall} w_{\sigma(i), j} \geq \mu_{i}\left(-q_{j, i}+q_{j, i+1}-\frac{f_{i}^{L(j)}}{\pi_{i}}\right) \\
& \underset{i}{\forall}-T^{L(i)}+u_{i}+\sum_{n \neq \sigma(i)} w_{n, i} \leq 0 \\
& \underset{L}{\forall} \in \sum_{i \in L} \sum_{L} \alpha^{L} f_{i}^{L} \leq-c^{L}, \\
& \underset{i, j}{\forall} q_{i, j}=q_{j, i} \\
& \underset{L}{\forall} \sum_{i \in L} \pi_{i}\left(q_{i, i}-q_{i, i+1}\right) \leq-c^{L} v \\
& \underline{T} \geq \sum_{L} \alpha^{L} T^{L} .
\end{aligned}
$$

Let $\bar{v}$ denote the value of the LP.

Then:

$$
T^{u}(N) \geq \frac{N}{N+\bar{v}} T
$$

These bounds can be used to study the behavior of loop interaction, priority interactions, and population level interactions in closed queuing networks with multiple roots. Two illustrative examples are provided below to illustrate the technique as well as illuminate some interesting phenomena in multi-loop systems. 


\section{Example 1.}

Consider the system shown in Figure B1, with the means of the service times $\vec{\mu}$ $=\{1 / 3,2 / 7,3 / 11,1,3,2\}$, and giving priority to buffers $b_{1}, b_{2}$ and $b_{3}$. Designate the loop containing buffers $b_{1}, b_{2}$ and $b_{3}$ as loop 1 or "the slower loop" due to its service rates. Using the theorems above, the infinite population throughput extrema are found to be the same $\left(\mathrm{T}^{*}=\underline{\mathrm{T}}\right)$ independent of the choice of loop population fraction $(\vec{\alpha})$ and weighting vector $(\vec{c})$ (see Figure B2). F urther, the throughput is invariant with changes in loop fraction population $(\vec{\alpha})$. However, plotting the asymptotic loss does show a variation with loop fraction population $(\vec{\alpha})$. These can be seen in Figure B3 showing $\underline{v}$, and Figure B4 showing $\bar{v}$.

Clearly, with buffers $b_{1}, b_{2}$ and $b_{3}$ having priority, the system favors the slow loop. As we increase the total population, we expect that the system will slow down as the fast loop stagnates for lack of service time. Figures B3 and B4 indicate that this transition takes place more slowly with increasing total population when the population distribution favors the faster loop.

\section{Example 2.}

Retaining the same system configuration from Figure B2, consider now the mean service times $\vec{\mu}=\{1 / 3,2,3 / 11,1,3,2 / 7\}$, and giving priority to buffers $b_{1}, b_{6}$, and $\mathrm{b}_{3}$. Figure B5 shows that $T^{*} \neq \underline{T}$ for every value of loop population fraction $(\vec{\alpha})$ and weighting factor $(\vec{c})$. This is not a trivial observation. Figure B 6 shows the summary of the throughputs obtained from one long simulation conducted at each of a large number of population levels, with population fractions fixed at ( $\vec{\alpha}=\{1 / 2,1 / 2\}$ ). An explanation for this intriguing graph may be the presence of two basins of attraction in the Markov state space. The communication rate between the two basins of attraction is extremely small so that once the system is captured by one of the two attractors, it stays there for the duration of the simulation. This result also demonstrates that the practice of "mixing" or taking an average of simulation results can be quite misleading in queuing applications. Finally, the result indicates that flow control schemes based on window size may succeed or fail based on the transient characteristics of the network and not steady-state behavior. 


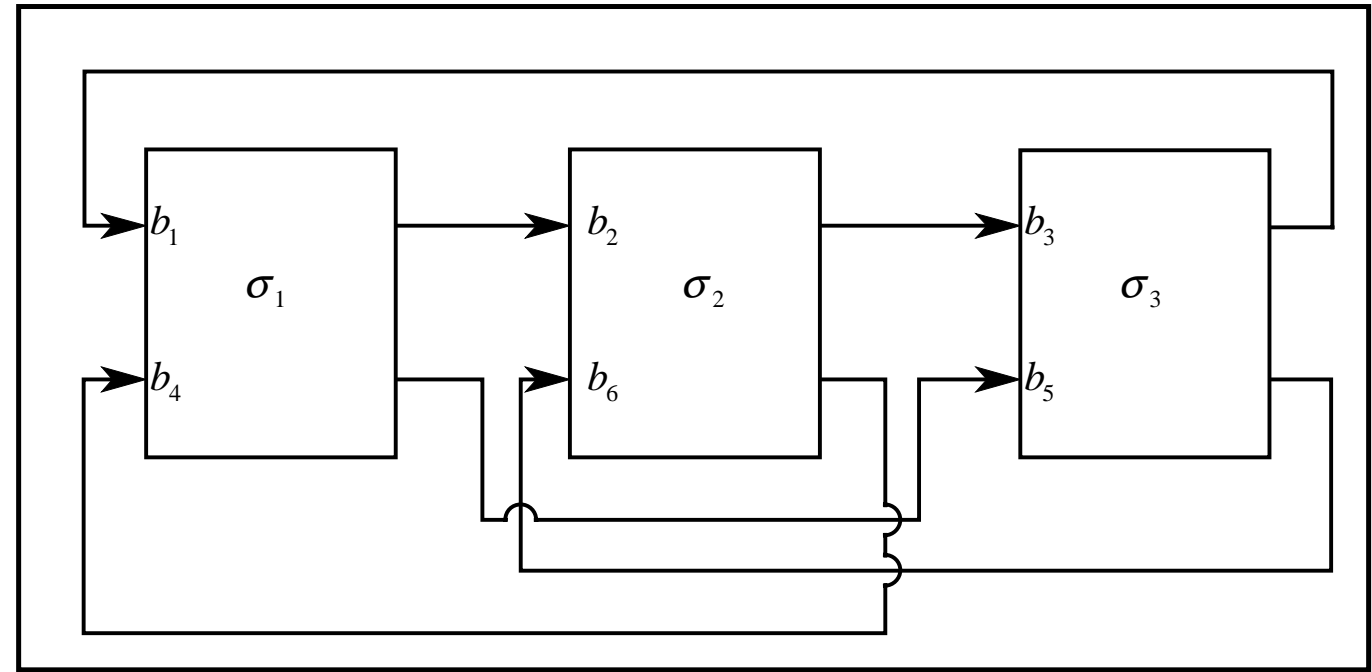

Figure B1. Multi-loop system (Ginsberg).

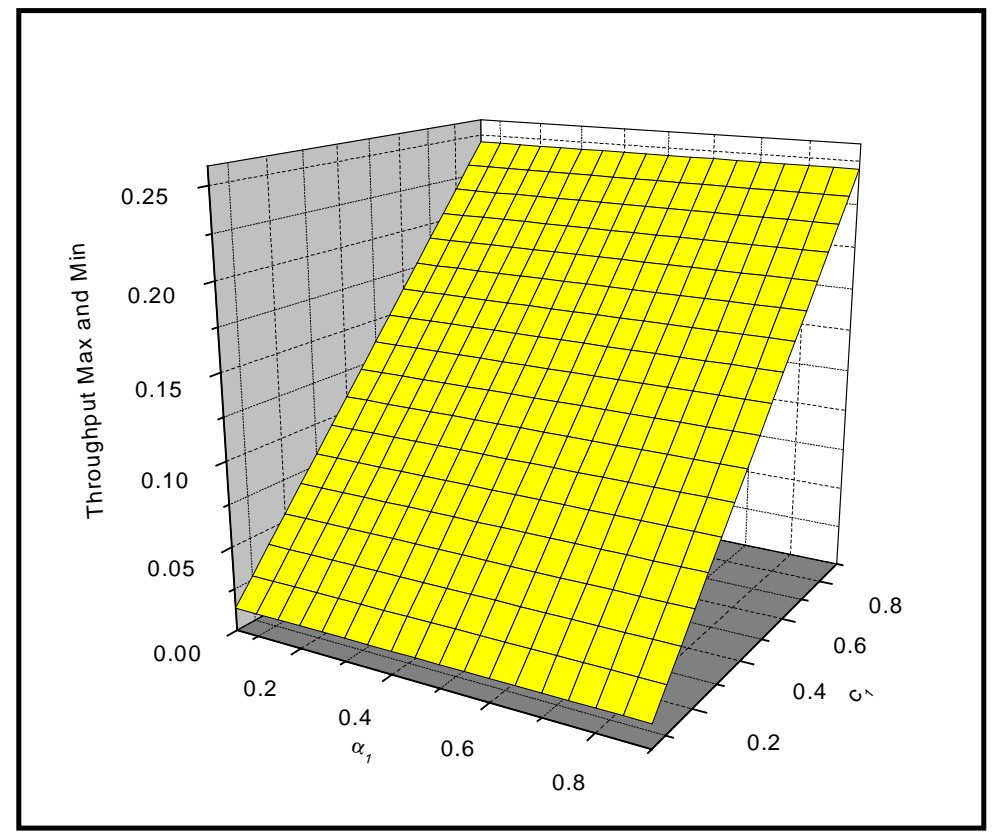

Figure B2. Throughput as a function of loop fraction population and weighting vector (Ginsberg). 


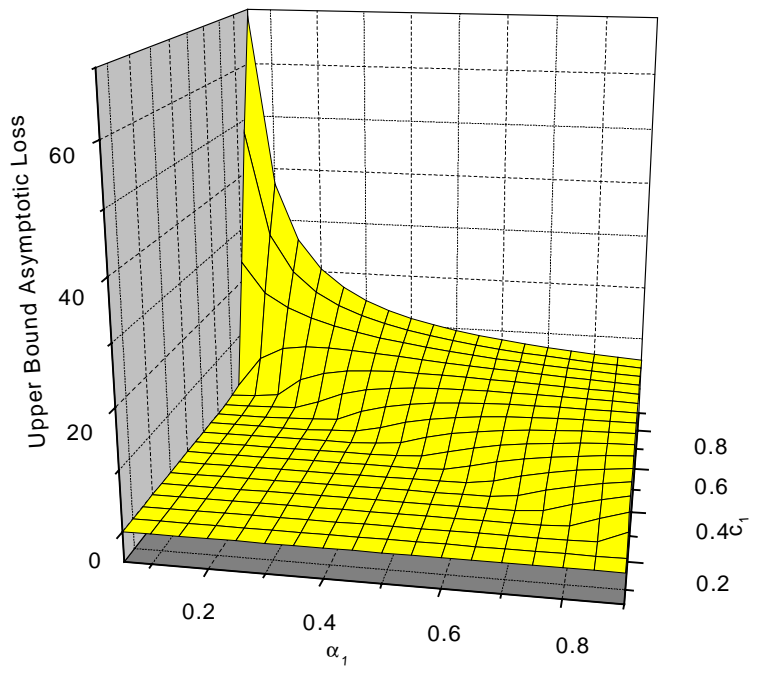

Figure B3. Lower bound asymptotic loss as a function of loop fraction population and weighting vector (Ginsberg).

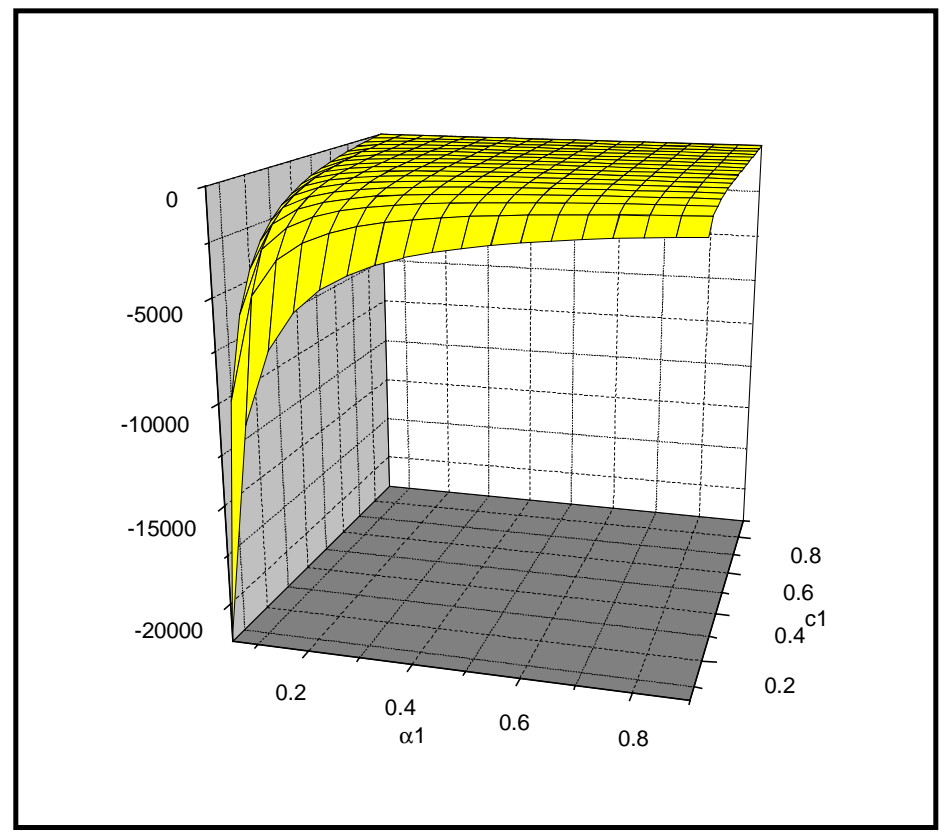

Figure B4. Upper bound asymptotic loss as a function of loop fraction population and weighting vector (Ginsberg). 


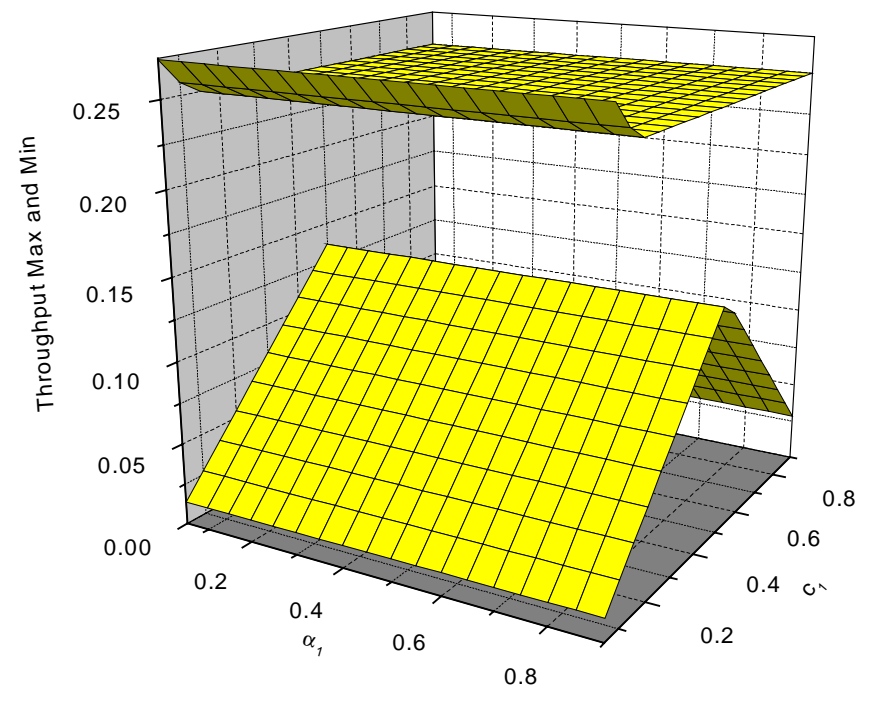

Figure B5. Theoretical bounds and simulation of throughput as a function of total population (Ginsberg).

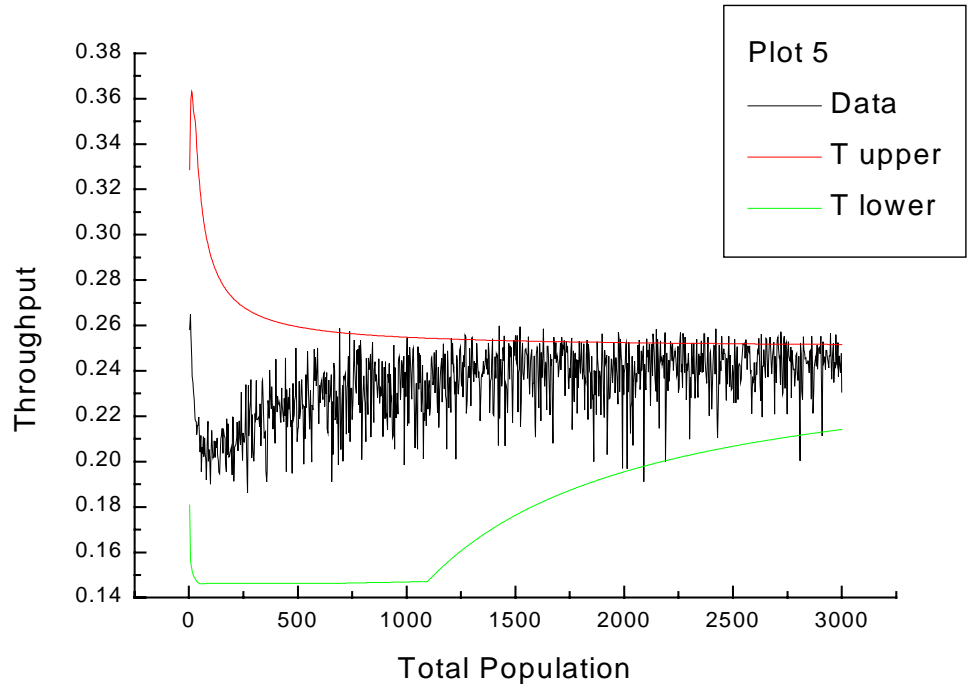

Figure B6. Summary of throughputs (Ginsberg). 


\section{Distribution}

Headquarters, Industrial Operations Command ATTN: HQIOC-EQC (2)

Headquarters, TACOM 48397-5000 ATTN: AMSTA-XE

Headquarters, FORSCOM

Forts Gillem \& McPherson 30330

$$
\text { ATTN: FCEN }
$$

Headquarters, TRADOC

Fort Monroe 23651

ATTN: ATBO-G

Chief of Engineers

ATTN: CEHEC-IM-LH (2)

ATTN: HECSA Mailroom (2)

ATTN: CECC-R

ATTN: CERD-L

ATTN: CERD-M

Defense Tech Info Center 22304

ATTN: DTIC-O (2) 
Public reporting burden for this collection of information is estimated to average 1 hour per response, including the time for reviewing instructions, searching existing data sources, gathering and maintaining the data needed, and completing and reviewing the collection of information. Send comments regarding this burden estimate or any other aspect of this collection of Information, including suggestions for reducing this burden, to Washington Headquarters Services, Directorate for Information Operations and Reports, 1215 Jefferson Davis Highway, Suite 1204, Arlington, VA 22202-4302, and to the Office of Management and Budget, Paperwork Reduction Project (0704-0188), Washington, DC 20503.
1. AGENCY USE ONLY (Leave Blank)
2. REPORT DATE
November 1999
3. REPORT TYPE AND DATES COVERED
Final

\begin{tabular}{|c|c|}
\hline $\begin{array}{l}\text { 4. TITLE AND SUBTITLE } \\
\text { Enhanced Operations and Maintenance of Pollution Control Equipment }\end{array}$ & $\begin{array}{l}\text { 5. FUNDING NUMBERS } \\
62720 \\
\text { D048 } \\
\text { U49 }\end{array}$ \\
\hline $\begin{array}{l}\text { 6. AUTHOR(S) } \\
\text { Jearldine I. Northrup, Mark D. Ginsberg, and Joyce C. Baird }\end{array}$ & \\
\hline $\begin{array}{l}\text { 7. PERFORMING ORGANIZATION NAME(S) AND ADDRESS(ES) } \\
\text { U.S. Army Construction Engineering Research Laboratory (CERL) } \\
\text { P.O. Box } 9005 \\
\text { Champaign, IL } 61826-9005\end{array}$ & $\begin{array}{l}\text { 8. PEFORMING ORGANIZATION } \\
\text { REPORT NUMBER } \\
\text { TR } 99 / 88\end{array}$ \\
\hline $\begin{array}{l}\text { 9. SPONSORING / MONITORING AGENCY NAME(S) AND ADDRESS(ES) } \\
\text { Headquarters, Industrial Operations Command (HQIOC) } \\
\text { ATTN: HQIOC-EQC } \\
\text { Rock Island, IL 61299-6000 }\end{array}$ & $\begin{array}{l}\text { 10. SPONSORING / MONITORING } \\
\text { AGENCY REPORT NUMBER }\end{array}$ \\
\hline
\end{tabular}

9. SUPPLEMENTARY NOTES

Copies are available from the National Technical Information Service, 5385 Port Royal Road, Springfield, VA 22161

12a. DISTRIBUTION / AVAILABILITY STATEMENT

12b.DISTRIBUTION CODE

Approved for public release; distribution is unlimited.

13. ABSTRACT (Maximum 200 words)

Laws and regulations mandate that Army installations monitor emissions from industrial processes, and maintain their processes within emissions standards. Army installations commonly use pollution control equipment (PCE) to monitor emissions, and to stay within regulatory and legal limits. Alternatives to use of PCE include using advanced technologies to detect problem conditions, collecting data to predict and determine the cause of failures, using dynamic modeling techniques to model the system or components that have a higher than expected frequency of failure, and verifying the efficacy of the model with data collected from test runs. This study summarized recent attempts to model large systems by using a model based on queuing theory. By studying the methods being used now for reliability centered maintenance (RCM) and the military's predilection to run-to-failure maintenance, this research has produced a linear program from the queuing model that can help reduce equipment downtime. These recent methods may allow U.S. Army installations to optimize maintenance policy for minimal ecological impact.

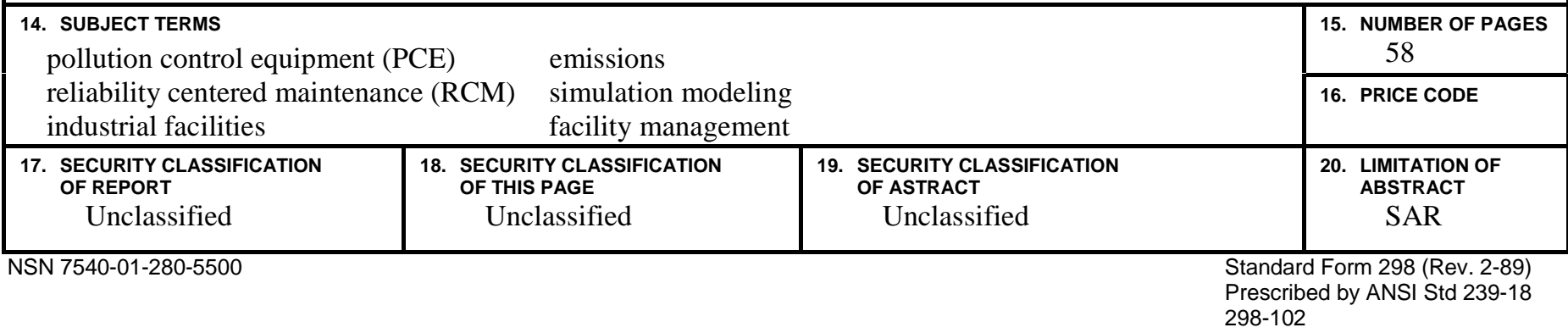

